\title{
Linearized semiclassical initial value time correlation functions with maximum entropy analytic continuation
}

\author{
Jian Liu and William H. Miller \\ Department of Chemistry and K. S. Pitzer Center for Theoretical Chemistry \\ University of California, \\ and Chemical Science Division, Lawrence Berkeley National Laboratory
}

Berkeley, California 94720-1460 


\begin{abstract}
The maximum entropy analytic continuation (MEAC) method is used to extend the range of accuracy of the linearized semiclassical initial value representation (LSC-IVR)/classical Wigner approximation for real time correlation functions. The LSC-IVR provides a very effective 'prior' for the MEAC procedure since it is very good for short times, exact for all time and temperature for harmonic potentials (even for correlation functions of nonlinear operators), and becomes exact in the classical high temperature limit. This combined MEAC+LSC/IVR approach is applied here to two highly nonlinear dynamical systems, a pure quartic potential in one dimensional and liquid para-hydrogen at two thermal state points (25K and $14 \mathrm{~K}$ under nearly zero external pressure). The former example shows the MEAC procedure to be a very significant enhancement of the LSC-IVR, for correlation functions of both linear and nonlinear operators, and especially at low temperature where semiclassical approximations are least accurate. For liquid para-hydrogen, the LSC-IVR is seen already to be excellent at $\mathrm{T}=25 \mathrm{~K}$, but the MEAC procedure produces a significant correction at the lower temperature $(T=14 \mathrm{~K})$. Comparisons are also made to how the MEAC procedure is able to provide corrections for other trajectory-based dynamical approximations when used as priors.
\end{abstract}




\section{Introduction}

Since classical molecular dynamics (MD) simulation methods are the only generally applicable approach for describing the dynamics of large molecular systems, we have been pursuing the use of various initial value representations (IVRs) $)^{1-17}$ of semiclassical (SC) theory ${ }^{18,19}$ to add quantum effects to classical MD simulations of time correlation functions. The SC-IVR provides a way for generating the quantum time evolution operator (propagator) $e^{-i \hat{i t t / \hbar}}$ by computing an ensemble of classical trajectories, much as is done in standard classical MD simulations. As is well known from SC developments in the early 1970's,18-21, such approaches actually contain all quantum effects at least qualitatively, and in molecular systems the description is usually quite quantitative (see reviews $^{2-5,14,22,23}$ and some recent applications $\left.{ }^{24-38}\right)$.

The simplest (and most approximate) version of the SC-IVR is its 'linearized' approximation (LSC-IVR) $^{6,24,25,34,39-44}$, which leads to the classical Wigner model ${ }^{45-48}$ for time correlation functions; see Section IIC for a summary of the LSC-IVR. The classical Wigner model is an old idea, but it is important to realize that it is contained within the SC-IVR approach, as a well-defined approximation to it $^{39,40}$. There are other ways to derive the classical Wigner model (or one may simply postulate it $)^{6,44,49,50}$, and we also note that the 'forward-backward semiclassical dynamics' (FBSD) approximation of Makri et al. ${ }^{24,28-30,51-62}$ is very similar to it. The LSC-IVR/classical Wigner model cannot describe true quantum coherence effects in time correlation functions-more accurate SC-IVR approaches, such as the Fourier transform forward-backward IVR (FB-IVR) approach ${ }^{22,63}$, or the still 
more accurate generalized FB-IVR ${ }^{64}$ and exact FB-IVR ${ }^{5}$ of Miller et al. , are needed for this-but it does describe some aspects of the quantum dynamics very well ${ }^{24-26,34-38,41,42,65-67}$. E.g., the LSC-IVR has been shown to describe the strong tunneling regime ${ }^{42}$ in reactive flux auto-correlation functions (which determine chemical reaction rates) quite well, and also velocity autocorrelation functions $^{24,25,66,67}$, force autocorrelation functions ${ }^{25,34-38}$, and incoherent dynamic structures ${ }^{26}$ in systems with enough degrees of freedom for quantum re-phasing to be unimportant.

The purpose of this paper is to describe use of the maximum entropy analytic continuation (MEAC) procedure ${ }^{68-84}$ to extend the accuracy of LSC-IVR time correlation functions. Recently Habershon et $a l^{84}$ showed that the ring polymer molecular dynamics (RPMD) model ${ }^{85-91,92}$ could provide a good 'prior' for the MEAC. LSC-IVR, RPMD, and the centroid molecular dynamics ${ }^{93-105}$ (CMD) are all approximate ways for adding some quantum effects to classical MD simulations, though none of them incorporate 'true' quantum coherence effects. Since the LSC-IVR approximation is more accurate in some aspects than RPMD and CMD (e.g., it is exact for harmonic systems for non-linear, as well as linear operators, also see Refs. ${ }^{26,106}$ ), it should thus provide an even more useful 'prior' for the MEAC methodology (the accuracy of which depends on having as good a 'prior' as possible). Since the LSC-IVR itself becomes accurate in the high-temperature and the harmonic limits (for both linear and nonlinear operators), the combination MEAC+LSC/IVR will obviously be good in both of these limits, and it should provide some improvement over the LSC-IVR description of strongly anharmonic systems and for lower temperatures. In addition, since the 
LSC-IVR includes some (approximate) real time dynamical information, the MEAC+LSC/IVR may be a good choice for systems involving a separation of time scales, where the MEAC with a featureless flat prior does not perform well ${ }^{107,108}$.

Section II briefly reviews the analytic relation between real time and imaginary time correlation functions $^{109}$, the MEAC procedure ${ }^{68-84}$ to utilize this relation efficiently, and the LSC-IVR methodology ${ }^{6,24,25,34,39-44}$. Section III then presents several applications to demonstrate how the MEAC+LSC/IVR approach performs: Sec IIIA demonstrates how the LSC-IVR prior is superior to the conventional flat prior in the harmonic limit and the high temperature regime, and Sec IIIB shows how the MEAC procedure improves the LSC-IVR for a strongly anharmonic one-dimensional model at very low temperature; and finally, Sec IIIC describes application of the MEAC+LSC/IVR to a complex system (liquid para-hydrogen) and compares the results with those using priors from other trajectory-based methods (RPMD, CMD, purely classical dynamics itself) with their MEAC corrections. Section IV summarizes and concludes.

\section{Theory and methodology}

\section{A. Analytic continuation between real time and imaginary time autocorrelation functions}

Most quantities of interest in the dynamics of complex systems can be expressed in terms of thermal time autocorrelation functions ${ }^{110}$, the standard version of which is

$$
C(t)=\left\langle\hat{A}(0) \hat{A}^{\dagger}(t)\right\rangle=\operatorname{Tr}\left(\hat{A}^{\beta} e^{i \hat{H} t / \hbar} \hat{A}^{\dagger} e^{-i \hat{H} t / \hbar}\right)
$$


where $\hat{A}^{\beta}=e^{-\beta \hat{H}} \hat{A} / Z$. Here $\hat{H}$ is the (time-independent) Hamiltonian for the system, which for large molecular systems is usually expressed in terms of its Cartesian coordinates and momenta

$$
\widehat{H}=\frac{1}{2} \hat{\mathbf{p}}^{\mathrm{T}} \mathbf{M}^{-1} \hat{\mathbf{p}}+V(\hat{\mathbf{x}})
$$

where $\mathbf{M}$ is the (diagonal) mass matrix, $(\hat{\mathbf{p}}, \hat{\mathbf{x}})$ the momentum and coordinate operators, respectively, $Z=\operatorname{Tr} e^{-\beta \hat{H}}\left(\beta=1 / k_{B} T\right)$ the partition function, and $\hat{A}$ the operator relevant to the specific property of interest, with $\hat{A}^{\dagger}$ its Hermitian conjugate. Its spectrum

$$
\underline{C}(\omega)=\int_{-\infty}^{\infty} d t e^{-i \omega t} C(t) \text { or } C(t)=\frac{1}{2 \pi} \int_{-\infty}^{\infty} d t e^{i \omega t} \underline{C}(\omega)
$$

is often experimentally measurable. It is easy to show (e.g., by working in the basis of eigenstates of $\hat{H})$ the non-negativity of $\underline{C}(\omega) \quad(\underline{C}(\omega) \geq 0)$ and the detailed balance relation

$$
\underline{C}(-\omega)=e^{-\beta \hbar \omega} \underline{C}(\omega)
$$

From the work by Baym and Mermin ${ }^{109}, C(t+i \hbar \lambda)$ is analytic within the strip $0 \leq \lambda \leq \beta$ in the complex plane. As a consequence, the correlation function $C(i \hbar \lambda)$ on the imaginary time axis contains the same physical information as and can in principle determine $C(t+i \hbar \lambda)$ within the strip $0 \leq \lambda \leq \beta$ by way of analytic continuation. The relation between the spectrum (Eq. (3)) and the imaginary time correlation function $C(i \hbar \lambda)$ is given by

$$
C(i \hbar \lambda)=\frac{1}{2 \pi} \int_{-\infty}^{\infty} d \omega e^{-\lambda \hbar \omega} \underline{C}(\omega)
$$

By the virtue of the detailed balance, i.e., Eq. (4), one has

$$
C(i \hbar \lambda)=\int_{0}^{\infty} d \omega K(\lambda, \omega) \underline{C}(\omega)
$$

where 


$$
K(\lambda, \omega)=\frac{1}{2 \pi}\left[e^{-\lambda \hbar \omega}+e^{-(\beta-\lambda) \hbar \omega}\right],
$$

which is a numerically well-behaved function of $\omega$ for any $\lambda(0 \leq \lambda \leq \beta)$. Based on Eq. (5),

similar relations between the imaginary-time autocorrelation function and other versions ${ }^{111,112}$ of real-time correlation function can be obtained, which are discussed in Appendix A.

\section{B. Maximum entropy analytic continuation (MEAC)}

Even for large systems, the imaginary time correlation function $C(i \hbar \lambda)$ can usually be calculated accurately by path integral techniques ${ }^{113,114}$ (with statistical noise). Techniques of numerical analytic continuation from the imaginary time autocorrelation function to the real time one include the Padé approximant scheme $\mathrm{e}^{115,116}$ and some procedures ${ }^{117}$ to solve the intrinsically unstable inverse problem of the integral equation, Eq. (6). Here we use the widely used maximum entropy analytic continuation (MEAC) method ${ }^{68-84}$ based on the Bayesian approach ${ }^{118,119}$. Followed is a brief summary of the MEAC procedure, while more details are described in above literature.

In the numerical version of Eq. (6), the frequency axis is discretized on a grid $\left\{\omega_{j}, j=1, \cdots, N\right\}$ with $A_{j}=\underline{C}\left(\omega_{j}\right) \Delta \omega_{j}$ where $\Delta \omega_{j}$ is the size of each grid spacing, and the imaginary time autocorrelation function $C_{k}=C\left(i \hbar \lambda_{k}\right)$ is known on a discrete set of points $\left\{\lambda_{k}, k=1, \cdots, M\right\}$, with $N$ usually being much large than $M$. Eq. (6) can then be expressed as

$$
\mathbf{C}=\mathbf{K A}
$$

where $\mathbf{A}$ and $\mathbf{C}$ are vectors with components $A_{j}$ and $C_{k}$ respectively, and $\mathbf{K}$ is the rectangular kernel matrix with elements $K_{k j}=K\left(\lambda_{k}, \omega_{j}\right)$ in Eq. (7). 
Since the imaginary time data $\left\{C_{k}\right\}$ for general systems obtained from path integral calculations typically contain inevitable statistical error, it is in general not best to seek a direct solution for $\left\{C_{k}\right\}$ in Eq. (8), e.g., by minimizing the standard mean squared deviation of the imaginary time data

$$
\chi^{2}(\mathbf{A})=(\mathbf{C}-\mathbf{K A})^{T} \mathbf{T}^{-1}(\mathbf{C}-\mathbf{K A})
$$

where $\mathbf{T}$ is the covariance matrix

$$
T_{i k}=\frac{1}{N_{m}\left(N_{m}+1\right)} \sum_{l=1}^{N_{m}}\left(\left\langle C_{i}\right\rangle-C_{i}^{(l)}\right)\left(\left\langle C_{k}\right\rangle-C_{k}^{(l)}\right)
$$

with $N_{m}$ being the number of statistically independent "measurements" from the path integral simulation and $\left\langle C_{k}\right\rangle$ denoting the average value of the measurements for $C_{k}=C\left(i \hbar \lambda_{k}\right)$. Instead, the MEAC method seeks a solution to the integral equation Eq. (8) which maximizes the function

$$
Q(\mathbf{A} ; \alpha)=\alpha S(\mathbf{A})-\chi^{2}(\mathbf{A}) / 2
$$

where the information entropy $S(\mathbf{A})$ is defined as

$$
S(\mathbf{A})=\sum_{j=1}^{N}\left[A_{j}-\stackrel{o}{A_{j}}-A_{j} \ln \frac{A_{j}}{A_{j}}\right]
$$

with $\stackrel{\circ}{A_{j}}=\stackrel{o}{\underline{C}}\left(\omega_{j}\right) \Delta \omega_{j}$ for a reasonable prior model for the spectrum $\stackrel{\circ}{\underline{C}}(\omega) ; S(\mathbf{A})$ thus quantifies how the final solution deviates from the prior model. The positive regularization parameter $\alpha$ in Eq. (11) weights the importance of the maximization of the information entropy $S(\mathbf{A})$ over the minimization of the standard mean squared deviation $\chi^{2}(\mathbf{A})$, i.e., a large value of $\alpha$ leads to a solution primarily determined by the maximization of $S(\mathbf{A})$, which is close to the prior model, while 
a small value of $\alpha$ gives a solution mostly determined from the minimization of $\chi^{2}(\mathbf{A})$, which gives a close fit to the imaginary time data (along with their statistical error).

For a given value of $\alpha$, maximizing $Q(\mathbf{A} ; \alpha)$ is accomplished by a numerically robust algorithm due to Bryan ${ }^{120}$ that has been commonly used in the MEAC literature above. Usually $Q(\mathbf{A} ; \alpha)$ is maximized for a series of $\alpha$ values in order to determine its best value. In this paper, we apply two standard techniques used in the MEAC literature for the choice of $\alpha$. One is the classic maximum entropy (CME) approach ${ }^{121}$, in which $\alpha$ is chosen to satisfy the Skilling equation

$$
-2 \alpha S(\mathbf{A})=\sum_{i} \frac{\varepsilon_{i}}{\alpha+\varepsilon_{i}} \equiv N_{g}
$$

where $\left\{\varepsilon_{i}\right\}$ are the eigenvalues of the curvature matrix of $\chi^{2}$

$$
\Lambda_{i j}=\frac{1}{2} \sqrt{A_{i}} \frac{\partial \chi^{2}}{\partial A_{i} \partial A_{j}} \sqrt{A_{j}}
$$

$N_{g}$, defined by Eq. (13), is often called the 'number of good data' or the number of effective independent measurements. The CME gives the optimum spectrum that simultaneously maximizes the posterior probabilities $\mathscr{P}(\mathbf{A} \mid \mathbf{C})$ for $\mathbf{A}$ given $\mathbf{C}$ and $\mathscr{P}(\alpha \mid \mathbf{C})$ for $\alpha$ given $\mathbf{C}$.

Another prescription for choosing $\alpha$ is the L-curve method ${ }^{122,123}$. In this procedure, after maximizing $Q(\mathbf{A} ; \alpha)$ for a succession of $\alpha$ values, a plot of $\log \left[\chi^{2}(\mathbf{A})\right]$ versus $\log [-S(\mathbf{A})]$ is constructed, and the resulting curve usually has a characteristic "L" shape. The horizontal leg of the " $L$ " curve represents a regime where any further deviation from the prior model brings little further benefit for accurate fitting of the imaginary time data. Therefore, the corner of the "L" curve, where the curvature of the curve is at a maximum, gives the optimum value of $\alpha$ that represents the 
best compromise between faithfully fitting the imaginary time data and keeping as close as possible to the prior model. It was pointed out by Krilov and Berne ${ }^{77}$ that if a flat (featureless) prior (MEAC-flat) is used, then the CME technique for choosing $\alpha$ is better for relatively sharp spectra, while the L-curve method gives more reliable results for a broad spectral distribution. In the applications described below, we compare these two techniques for implementing the MEAC using various priors, e.g., that afforded by the LSC-IVR.

\section{Linearized semi-classical initial value representation (LSC-IVR)}

The SC-IVR approximates the forward (backward) time evolution operator $e^{-i \hat{H} t / \hbar}\left(e^{i \hat{H} t / \hbar}\right)$ by a phase space average over the initial conditions of forward (backward) classical trajectories ${ }^{2,4,5,18}$. By making the (drastic but reasonable) approximation that the dominant contribution to the phase space averages comes from forward and backward trajectories that are close to one another and then linearizing the forward and backward actions of such trajectories, Miller and coworkers ${ }^{39-41}$ obtained the linearized SC-IVR (LSC-IVR), or classical Wigner model for a general A-B correlation function (i.e., replacing the operator $\hat{A}^{\dagger}$ by $\hat{B}$ in Eq. (1)),

$$
C_{A B}^{L S C-I V R}(t)=(2 \pi \hbar)^{-3 N_{p}} \int d \mathbf{x}_{0} \int d \mathbf{p}_{0} A_{w}^{\beta}\left(\mathbf{x}_{0}, \mathbf{p}_{0}\right) B_{w}\left(\mathbf{x}_{t}, \mathbf{p}_{t}\right)
$$

where $A_{w}^{\beta}$ and $B_{w}$ are the Wigner functions ${ }^{45}$ corresponding to these operators,

$$
O_{w}(\mathbf{x}, \mathbf{p})=\int d \mathbf{\Delta} \mathbf{x}\langle\mathbf{x}-\Delta \mathbf{x} / 2|\hat{O}| \mathbf{x}+\Delta \mathbf{x} / 2\rangle e^{i \mathbf{p}^{T} \Delta \mathbf{x} / \hbar}
$$

for any operator $\hat{O}$. Here $N_{p}$ is the number of particles in the system, and $\left(\mathbf{x}_{0}, \mathbf{p}_{0}\right)$ is the set of initial conditions (i.e., coordinates and momenta) for a classical trajectory, $\left(\mathbf{x}_{t}\left(\mathbf{x}_{0}, \mathbf{p}_{0}\right), \mathbf{p}_{t}\left(\mathbf{x}_{0}, \mathbf{p}_{0}\right)\right)$ 
being the phase point at time $t$ along that trajectory. (More recently, Liu and Miller ${ }^{6}$ have shown that the exact quantum time correlation function can be expressed in the same form as Eq. (15), with an associated dynamics in the single phase space, and it was furthermore demonstrated that the LSC-IVR is its classical limit ( $\hbar \rightarrow 0$ ), high temperature limit ( $\beta \rightarrow 0$ ), and harmonic limit.) The LSC-IVR can be applied not only to correlation functions at equilibrium but also to non-equilibrium correlation functions. These merits of the LSC-IVR make it a versatile tool to study quantum-mechanical effects in chemical dynamics of large molecular systems.

A major shortcoming of the LSC-IVR, however, is that the quantum mechanical equilibrium distribution is not correctly conserved-i.e., for the case $\hat{A}=1$ the correlation function (i.e. the canonical ensemble average of operator $\hat{B}$ ) is not time-independent. The degree to which this affects the accuracy of the LSC-IVR in practical applications is subtle, since the LSC-IVR quantizes $\hat{A}^{\beta}$ rather than the Boltzmann density operator $e^{-\beta \hat{H}} / Z$. A previous investigation ${ }^{26}$ suggested that this inconsistency in the LSC-IVR correlation function may be most noticeable at very long time for some systems at quite low temperature.

The relation between the real time and imaginary time correlation functions, i.e., Eq. (6), provides a way to check the behavior of the LSC-IVR. Furthermore, considering the merits of the LSC-IVR, using it as the prior in the MEAC approach should certainly be better than using a featureless flat prior. We now investigate how the MEAC behaves using the LSC-IVR prior (MEAC+LSC/IVR). 


\section{Results and discussions}

In all calculations below, we have used the bisection method ${ }^{113}$ of the path integral Monte Carlo (PIMC) approach to calculate the imaginary time correlation function $C(i \hbar \lambda)$. The LSC-IVR results for real time correlation functions were obtained with the thermal Gaussian approximation (TGA) ${ }^{124-129}$ (TGA/LSC-IVR) that we have implemented recently ${ }^{6,24-26}$. The MEAC procedure for the standard version of the correlation function uses only its real part for convenience and involves Eqs. (A.3)-(A.4), and that for the Kubo-transformed version utilizes Eqs. (A.9)-(A.10). The flat prior for the standard version of the correlation function is

$$
\stackrel{o}{C}^{R}(\omega)=\left\{\begin{array}{l}
\pi C^{R}(t=0) / \omega_{\max }, \quad|\omega| \leq \omega_{\max } \\
0, \quad \text { otherwise }
\end{array}\right.
$$

and that for the Kubo-transform version is

$$
\underline{o}^{\text {kubo }}(\omega)=\left\{\begin{array}{l}
\pi C^{k u b o}(t=0) / \omega_{\max }, \quad|\omega| \leq \omega_{\max } \\
0, \text { otherwise }
\end{array}\right.
$$

Here $\left[-\omega_{\max }, \omega_{\max }\right]$ is the range of the frequency that covers all significant parts of the spectrum.

Eq. (17) or (18) gives the correct initial value of the time correlation function as a constraint.

\section{A. Advantages of the LSC-IVR prior}

The MEAC using the flat prior usually fails when the spectrum has multiple maxima, and when the imaginary time data are limited and noisy. The former usually happens when the operator $\hat{A}$ is a highly nonlinear operator, and the latter occurs for high temperature (i.e., when $\hbar \beta \rightarrow 0$ ). The LSC-IVR, on the other hand, treats nonlinear and linear operators to an equivalent level of 
accuracy $^{26}$ and naturally has the correct (i.e., classical) limit for high temperature.

The elementary (but important) case of a harmonic potential,

$$
V(x)=\frac{1}{2} m \omega^{2} x^{2}
$$

provides a dramatic illustration of these features (where for simplicity we take $m=1, \omega=1$ and $\hbar=1$ ). $\quad$ In this case the LSC-IVR itself (i.e., without any MEAC correction) gives exact results for the autocorrelation function for both nonlinear and linear operators, for arbitrarily long time and for any temperature, so here we are interested in showing how the MEAC procedure behaves if one uses the flat prior. Choosing a highly nonlinear operator $\hat{A}=\exp \left[-5 \hat{x}^{2}\right]$ and the inverse temperature $\beta=5,32$ beads were used for the PIMC calculation of the imaginary time correlation function. Both the CME and the L-curve techniques were used for the MEAC with the flat prior spectrum, with the maximal frequency $\omega_{\max }=25$ in Eq. (17). Although Figure 1 shows that the MEAC with the flat prior fits the imaginary time data very well, the real time correlation function which results from it is seen in Figure 2 to behave poorly-it only has reasonable agreement with the exact result for times shorter than $0.1 \hbar \beta$ and fails to capture the (classical) coherence that occurs on the thermal time scale $\hbar \beta$. Since the exact spectrum contains a set of delta functions $\delta(2 j \omega)(j=0, \pm 1, \pm 2, \cdots)$, it is perhaps not surprising that the MEAC with a prior that is not reasonably close to the exact result will encounter severe problems. This example reveals that the MEAC is not guaranteed to give the result correct to the order of the thermal time $\hbar \beta$, and that a good prior is important for the MEAC procedure when the operator involved in the correlation 
function is highly nonlinear. For near-harmonic potentials, the LSC-IVR does a quite good job for all kinds of operators ${ }^{6,24}$ and thus probably provides the best prior.

The next example is more challenging, a purely quartic potential,

$$
V(x)=\frac{1}{4} x^{4}
$$

with $m=1$ and $\hbar=1$. Here, of course, the LSC-IVR is not exact for this highly anharmonic potential: Figure 3 shows that it is able to produce the first one or two oscillations in the standard momentum and Kubo-transformed force autocorrelation functions, but it de-phases for longer time, being unable to describe the purely quantum coherent oscillations at longer time. (To capture these one would need to go to more accurate levels of semiclassical theory for the correlation function ${ }^{5,64}$, but we note that in many cases in complex systems one expects such long time coherent effects to be quenched by coupling among the various degrees of freedom ${ }^{2,130,131}$.) Nevertheless, Figure 3 shows that the LSC-IVR correlations functions are considerably better than ones given by the MEAC using a flat prior in Eq. (17) and Eq. (18), respectively, with $\omega_{\max }=8$ at a relatively high temperature $\beta=1$. 8 beads were used for the PIMC calculation of the imaginary time data. The MEAC works somewhat better for the case of linear operators [Figure 3(a)] compared to non-linear ones [Figure 3(b)], but not nearly so well as the uncorrected LSC-IVR. This again suggests that the LSC-IVR will provide a much more effective prior for use in the MEAC procedure, for both nonlinear and linear operators, and we now describe this behavior for the quartic potential.

\section{B. MEAC with the LSC-IVR prior}


The exactness of the LSC-IVR in the harmonic limit, the classical limit, and the high-temperature limit, and the ability to treat both linear and nonlinear operators well, suggests that it should be very effective for use as a prior in the MEAC procedure. Compared with the featureless flat prior, it provides some (approximate) real time dynamical information as input to the MEAC. The MEAC+LSC/IVR will thus work well in the three limits described above (since the LSC-IVR, the prior, does well all by itself) and should work better than the original LSC-IVR for quite anharmonic systems in the very low temperature regime by virtue of the constraint from the MEAC procedure.

Since the LSC-IVR already does a good job for near-harmonic potentials, we focus on the quartic potential of Eq. (20) to provide a more meaningful test of the methodology. High and low temperature, $\beta=1$ and $\beta=10$, are considered, with 8 and 64 beads used respectively in the bisection method to compute the imaginary time correlation functions. Both the standard momentum and the Kubo-transformed force autocorrelation functions were computed via the MEAC+LSC/IVR procedure. At high temperature, $\beta=1$, the noticeably better fit of the imaginary time data due to the MEAC procedure, as seen in Figures 4, does NOT cause noticeable improvement in the real time LSC-IVR correlation functions shown in Figures 5. This is not a surprise, for the analysis in Appendix A shows that the MEAC procedure does little to improve the real time results for times much larger than the thermal time $\hbar \beta$. On the other hand, at the low temperature, $\beta=10$, the more subtle improvement for the fit of the imaginary time data, as shown in Figure 6, significantly 
improves LSC-IVR real time correlation functions, shown in Figure 7. This is of course due to the fact that the MEAC procedure works better when the thermal time $\hbar \beta$ is larger, for there is more statistically independent imaginary time data. It is quite encouraging to see in Figure 7 how much the MEAC+LSC/IVR approach improves over the original LSC-IVR correlation function in both the amplitude and the frequency of the oscillations at low temperature. For this strongly anharmonic potential, however, the MEAC+LSC/IVR still has the de-phasing behavior for times larger than $\hbar \beta$, which is more noticeable for the nonlinear operator [Figure 7(b)].

Since the spectrum of this one-dimensional model potential contains sharp peaks, the results in Figure 7 agree with the observation by Krilov and Berne ${ }^{77}$ that in this situation the CME is indeed a better choice than the L-curve method, also for the better LSC-IVR prior. These results also demonstrate that the MEAC procedure works better for linear operator than for nonlinear ones.

\section{Application to liquid para-hydrogen}

Although $\mathrm{H}_{2}$ is the lightest and thus most quantum-like molecule, quantum effects due to exchange of identical molecules are negligible in its liquid phase. Liquid para-hydrogen is well described by the Silvera-Goldman (SG) model $^{132}$, an isotropic pair potential in which the para-hydrogen molecule is treated as a sphere particle. (The spherical approximation is known to be accurate because the temperature of liquid para-hydrogen is much too low for any rotational state other than $\mathrm{J}=0$ to be populated.) Liquid para-hydrogen has served as a benchmark system to test many approximate quantum dynamic methods, e.g., the MEAC with the flat prior $^{82}$, quantum 
mode-coupling theory $(\mathrm{QMCT})^{133}$, the $\mathrm{CMD}^{102,134,135}$, the $\mathrm{RPMD}^{89,135}$, the $\mathrm{FBSD}^{28}$, and the LSC-IVR $^{25,65}$. Recently we have calculated the Kubo-transformed momentum autocorrelation function using the TGA/LSC-IVR for liquid para-hydrogen at two state points $T=25 \mathrm{~K}, v=31.7 \mathrm{~cm}^{3} \mathrm{~mol}^{-1}$ and $T=14 \mathrm{~K}, v=25.6 \mathrm{~cm}^{3} \mathrm{~mol}^{-1}$; details of these simulations are described in our previous work ${ }^{25}$.

The Kubo-transformed momentum autocorrelation function for this system thus serves as an excellent benchmark also for testing how well the MEAC procedure extends the accuracy of the LSC-IVR and also how it compares in this regard to using other approximate quantum dynamics methods (RPMD and CMD), and also classical dynamics itself, as the prior. (Note that all these trajectory-based methods give the exact initial value, i.e., $C_{p p}^{k u b o}(t=0)=3 m / \beta$, which is a good starting point for comparison.) For the PIMC calculation of the imaginary time correlation functions below, 32 beads were used for the state point at $T=25 \mathrm{~K}$ and 64 beads for that at $T=14 \mathrm{~K}$. For the MEAC procedure, we used $\omega_{\max } \approx 124 \mathrm{ps}^{-1}$ in Eq. (18). For the broad frequency spectrum of the correlation function for this system (see below), we observe that the L-curve technique produces smoother and more reasonable results than the CME when the flat prior is used (as mentioned in Ref. ${ }^{77}$ ), but interestingly there is very little difference between these two ways of choosing the regularization parameter $\alpha$ when the prior is given by any trajectory-based method; thus we will simply show the results based on the L-curve technique for the flat prior and will not distinguish between the 'MEAC-CME' and 'MEAC-L' for priors obtained from trajectory-based methods. 
First, we are able to check the accuracy of the various priors themselves for pure imaginary time data by Fourier transforming the real time correlation functions given by the four trajectory-based methods (LSC-IVR, RPMD, CMD, and classical mechanics itself) to obtain the spectra, and then using Eq. (A.9) to obtain the imaginary time correlation function. Figure 8 shows the imaginary time correlation functions for both state points: the exact (PIMC) result (solid circles) is compared to the results for the four trajectory-based priors that we consider. One sees that the LSC-IVR result is in very good agreement with the exact imaginary time values, with the CMD ${ }^{102}$ further off and the RPMD ${ }^{84}$ even more so, with the classical result deviating the most. Thus even though both the CMD and the RPMD models conserve the Boltzmann distribution in extended phase spaces, this property does not guarantee that they reflect the true quantum dynamics better.

When we apply the MEAC procedure for these priors, we find that the information entropies as defined in Eq. (12) (with the optimal value of $\alpha$ for each prior) lie in the order $S_{\text {flat }} \ll S_{\text {classical }}<S_{R P M D}, S_{C M D}, S_{L S C-I V R}<0$ at both state points (which is consistent with observations by Habershon et $a l^{84}$ ). Since the more accurate the prior, the closer the information entropy is to zero, this suggests that the approximate quantum dynamic methods (LSC-IVR, RPMD and CMD) indeed provide better priors, and even the classical prior is probably superior to the featureless flat prior, especially so the higher the temperature.

Figures 9 and 10 show the spectra (i.e., the Fourier transform of the Kubo-transformed real time correlation function) for the $25 \mathrm{~K}$ and $14 \mathrm{~K}$ state points, respectively. First, Figure 9(a) shows 
the spectra given by the four trajectory-based dynamics methods (LSC-IVR, RPMD, CMD, and classical), which serve as the priors for the MEAC procedure. Though qualitatively similar, one sees that there are significant variations. Figure 9(b), however, shows that once the MEAC procedure is applied, all four methods give essentially same result, except for some differences at very low frequency. (The MEAC+RPMD results here and below are directly from Ref. ${ }^{84}$, but MEAC results with all other priors are computed with imaginary time data in this paper.) Also shown in Figure 9(b) is the result of using the flat prior, and it is seen to differ significantly from those given by the MEAC using priors that contain dynamical information.

The good agreement of the MEAC-corrected results in Figure 9(b) using the four different trajectory-based priors is reassuring, but it is illuminating to look in more detail at the MEAC correction for each case; Figures 9(c-f) thus show the spectra given by the trajectory-based prior itself, compared to the MEAC-corrected result, for each of the four trajectory-based priors. The most important observation seen here (cf. Figure 9(c)) is that the MEAC procedure provides essentially no correction to the LSC-IVR prior, giving even greater confidence that it is close to the correct result. The MEAC corrections for the other three trajectory based priors are seen (Figures 9(d-f)) to be more significant, but obviously not so large in this case as to cause significant error since all four MEAC-corrected results are in reasonably good agreement (Figure 9(b)). (This result could have perhaps been anticipated from the results in Figure 8(a), namely that the LSC-IVR gives an imaginary time correlation function in better agreement with the exact result than the other approximate 
dynamical methods.)

The lower temperature results shown in Figure 10 are quite a different story: Figure 10(a) shows the spectra given by the four trajectory-based priors, and the differences between them are much greater than for the higher temperature case. The four MEAC-corrected spectra (and also the MEAC result with a flat prior) are shown in Figure 10(b) and though the difference between them all has been greatly reduced by the MEAC procedure, the differences are still quite noticeable. Again, Figures 10(c-f) show the spectra given by each of the priors, compared to the MEAC-corrected result, again emphasizing the large correction made by the MEAC procedure. It seems clear that the MEAC procedure definitely improves the results for each of the four trajectory-based methods, but the corrections are so large that it is difficult to know which of the MEAC-corrected results in Figure 10(b) are more accurate.

In Figures 10(c-f), although the change of the position of the maximum in the spectrum between the LSC-IVR and its MEAC correction looks more dramatic than that between the CMD (or RPMD) and its correction, the information entropies which measure the overall adjustment of the spectra suggest that the LSC-IVR is not necessarily worse than the CMD (or RPMD) at such low temperature (i.e., $S_{C M D}=-3.39 \AA^{\circ} \mathrm{ps}^{-2}<S_{L S C-I V R}=-2.11 \AA^{\circ} \mathrm{ps}^{-2}$ ). That the TGA/LSC-IVR result at 14K [Figure 10(c)] does not behave as well as that at $25 \mathrm{~K}$ [Figure 9(c)] may partly be due to the fact that the thermal Gaussian approximation (TGA) ${ }^{124-129}$ that we use to represent the Boltzmann operator $^{6,24-26}$ is a type of local harmonic approximation, which is less accurate for obtaining the 
Wigner transform $A_{w}^{\beta}$ at lower temperature. (In fact, all other local harmonic approximations $s^{34,44}$ encounter this problem.)

Turning now to the real time correlation functions themselves, Figures 11 and 12 show the results for the higher and lower temperature cases, respectively, and they mirror the behavior seen above for the spectra. First for the higher temperature, Figure 11(a) shows the real time correlation functions (corresponding to the spectra in Figure 9(a)) from the four trajectory-based methods themselves, and they show significant differences. However, the MEAC-corrected correlation functions shown in Fig 11(b) (corresponding to the spectra in Figure 9(b)) are in very good agreement with each other (except for the result obtained using the flat prior that is also shown in Figure 9(b)). Figures 11(c-e) show more detailed comparisons, specifically the LSC-IVR (and its MEAC-corrected version) compared to each of the other three trajectory based correlation functions (and their MEAC-corrected versions). Here one sees that the MEAC procedure provides essentially no correction in the LSC-IVR case (just as for the case of the spectra), and that the primary region in which the other three trajectory based methods are in error (before the MEAC correction) is a too rapid fall off at short time.

The lower temperature case is again more challenging. Figure 12(a) shows the real time correlation functions given by the four trajectory-based methods: the RPMD and CMD results agree well with each other, and also reasonably well with the classical, all three having prominent minima at $\sim 0.2$ to 0.3 ps. The LSC-IVR correlation function, on the other hand, has only a very shallow 
minimum at this time. Once corrected by the MEAC procedure, however, Figure 12(b) shows that all four results—and even a fifth one corresponding to the flat prior-are in much better agreement. However, there are noticeable differences between all of the results in the region beyond the minimum. It is difficult at present to know which of these results is more accurate in this regime; more accurate calculations for long time will be required to clarify this.

As a final quantitative test, diffusion constants have been computed from the real time correlation functions,

$$
D=\frac{1}{3 m^{2} N_{p}} \int_{0}^{\infty} d t C_{p p}(t)=\frac{1}{6 m^{2} N_{p}} \underline{C}_{p p}(\omega=0)
$$

at the two state points for the four trajectory-based dynamical approximations, along with the MEAC results obtained using these as the priors (and also that given by using the flat prior); these results are listed in Tables 1-2. At both temperatures the MEAC procedure considerably narrows the spread in values given by the various dynamical methods, the more so for the lower temperature, where variations of more than a factor of 2 are reduced to 20 30\%. The MEAC+LSC/IVR gives nearly the same result as the LSC-IVR at $T=25 \mathrm{~K}$, but for the lower temperature the MEAC procedure decreases it by $\sim 25 \%$ and brings it into much better agreement with experiment. The results in Tables 1-2 suggest that the MEAC procedure is very likely an improvement for all other priors as well ${ }^{136}$.
IV. Conclusions 
By using the LSC-IVR as the prior for the maximum entropy analytic continuation (MEAC)

procedure, we have shown that the MEAC+LSC/IVR approach significantly improves the region of accuracy of the LSC-IVR approximation for real time correlation functions. This is particularly useful for low temperature, where a semiclassical approximation such as the LSC-IVR is expected to be least accurate on its own. The MEAC correction to the LSC-IVR is effective for correlation functions of both linear and non-liner operators. This approach has been demonstrated for both a challenging one-dimensional model (a pure quartic potential) and a realistic system (liquid para-hydrogen at two state points). This MEAC extension of the LSC-IVR approximation should be of use in a variety of other applications, e.g., for evaluating the correlation functions relevant to vibrational energy relaxation ${ }^{34-38}$ in liquids.

The application to liquid para-hydrogen showed that other trajectory-based quantum dynamics approximations (RPMD ${ }^{84}, \mathrm{CMD}$ and classical dynamics itself) also benefit from MEAC corrections, and furthermore the differences between the correlation functions given by the various dynamics methods are considerably reduced by the MEAC correction process. Use of the classical correlation function itself as the prior, i.e., the MEAC+classical approach, was seen to work fairly and suggests an extremely economic way to obtain a rough estimate of quantum dynamical effects in the correlation functions for condensed matter systems ${ }^{137}$. (We also note that for all the priors containing dynamic information for the system in the MEAC procedure, the CME seems to work as well as the L-curve technique even when the spectrum is broad and work better when the spectrum contains sharp 
peaks, and thus seems to be a viable choice for us.)

Though the MEAC+LSC/IVR approach extends the time scale over which quantum coherence behavior can be correctly described, it nevertheless does de-phase at still longer times. In order to capture true quantum interference effects in time correlation functions for longer time, the improved version of the LSC-IVR ${ }^{6}$ and more accurate SC-IVR approaches (such as the generalized FB-IVR ${ }^{64}$ and exact FB-IVR ${ }^{5}$ of Miller et al.) are needed, e.g., for use as better priors in the MEAC procedure. Further investigation along such directions is certainly warranted.

\section{Acknowledgement}

This work was supported by the Office of Naval Research Grant No. N00014-05-1-0457 and

by the Director, Office of Science, Office of Basic Energy Sciences, Chemical Sciences, Geosciences, and Biosciences Division, U.S. Department of Energy under Contract No. DE-AC02-05CH11231. We also acknowledge a generous allocation of supercomputing time from the National Energy Research Scientific Computing Center (NERSC). We thank F. Paesani and G. Voth for providing the CMD data in Ref ${ }^{102}$. J. L. thanks C. Predescu for a discussion on the bisection method ${ }^{113}$ of the PIMC about two years ago and also thanks T.F. Miller for some useful discussions. 
Appendix: More discussion on the relation between real time and imaginary time autocorrelation functions

For the standard version of correlation function given by Eq. (1), it can be verified that

$$
C^{*}(t)=C(-t)
$$

so that by virtue of detail balance, Eq. (4), the spectrum defined in Eq. (3) can be obtained from the real part of $C(t)$ (which usually is more convenient),

$$
\underline{C}(\omega)=\frac{2}{1+e^{-\beta \hbar \omega}} \int_{-\infty}^{\infty} d t e^{-i \omega t} \operatorname{Re}[C(t)] \equiv \frac{2}{1+e^{-\beta \hbar \omega}} \underline{C}^{R}(\omega)
$$

Eq. (6) then has the equivalent form

$$
C(i \hbar \lambda)=\int_{0}^{\infty} d \omega K^{R}(\lambda, \omega) \underline{C}^{R}(\omega)
$$

where

$$
K^{R}(\lambda, \omega)=\frac{1}{\pi}\left[\frac{e^{-\lambda \hbar \omega}+e^{-(\beta-\lambda) \hbar \omega}}{1+e^{-\beta \hbar \omega}}\right]
$$

which is also a function which exponentially decays as $\omega \rightarrow \infty$ for any $\lambda(0<\lambda<\beta)$ and is constant for $\lambda=0, \beta$. Substituting Eq. (A.2) into Eq. (A.3) leads to the following relation between the imaginary and real time correlation functions,

$$
C(i \hbar \lambda)=\int_{0}^{\infty} d t g^{R}(\lambda, t) \operatorname{Re}[C(t)]
$$

where 


$$
\begin{aligned}
g^{R}(\lambda, t) & =\int_{0}^{\infty} d \omega e^{-i \omega t} K^{R}(\lambda, \omega) \\
& =\left\{\begin{array}{c}
\frac{1}{\hbar \beta} \operatorname{Im}\left[\frac{1}{\sinh (\pi t / \hbar \beta-i \pi \lambda / \beta)}-\frac{1}{\sinh (\pi t / \hbar \beta+i \pi \lambda / \beta)}\right] \\
\delta(t) \quad(\text { for } \lambda=0, \beta)
\end{array}\right.
\end{aligned}
$$

One can verify that $g^{R}(\lambda, t)=g^{R}(\beta-\lambda, t)$. Figure 13 plots $g^{R}(\lambda, t)$ as a function of $t / \hbar \beta$. It is clear from Eq. (A.6) and Figure 13 that the imaginary time data $C(i \hbar \lambda)$ are more sensitive to the real time data at short times (on the order of $\hbar \beta$ ) rather than long times, or inversely that the influence of the imaginary time data on the real time correlation function decays exponentially with time. In particular, for $\lambda=0$ or $\beta, g^{R}(\lambda, t)$ becomes a delta function, meaning that the correlation function at these values of imaginary time is fully determined by the initial $(t=0)$ value of $C(t)$; and when $\lambda=\beta / 2, g^{R}(\lambda, t)$ has the longest tail, meaning that $C(i \hbar \beta / 2)$ is the imaginary time correlation which affects the correlation function for longest values of real time, and thus has the strongest influence on long time limit properties such as reaction rates or diffusion constants in the analytic continuation procedure.

Since the integral of $g^{R}\left(\lambda, t^{\prime}\right)$ has is normalized to unity, i.e., $\int_{0}^{\infty} d t^{\prime} g^{R}\left(\lambda, t^{\prime}\right)=1$, one can define a function

$$
G^{R}\left(\lambda ; t_{1}, t_{2}\right)=\int_{t_{1}}^{t_{2}} d t^{\prime} g^{R}\left(\lambda, t^{\prime}\right)
$$

as a measure of the weight contributed from the real part of the real time correlation function between the time interval $\left[t_{1}, t_{2}\right]$ to the imaginary time data $C(i \hbar \lambda)$ and vice versa; note that 
$G^{R}\left(\lambda ; t_{1}, t_{2}\right)=G^{R}\left(\beta-\lambda ; t_{1}, t_{2}\right) \in[0,1]$. Figure 14 plots $G^{R}\left(\lambda ; t_{1}, t_{2}\right)$ as a function of $\lambda / \beta$. Again, one sees that values of the correlation function around the imaginary time $\lambda=\beta / 2$ constrain the longest real time value of the correlation function, and that analytic continuation from the imaginary time data $C(i \hbar \lambda)$ [such as Eq. (A.3) or (A.5)] provides little information on real times longer than the order of the thermal time $\hbar \beta$.

Finally, in addition to the standard autocorrelation function, there are other versions of the autocorrelation function, such as the symmetrized version ${ }^{111}$ with $\hat{A}_{s y m}^{\beta}=e^{-\beta \hat{H} / 2} \hat{A} e^{-\beta \hat{H} / 2} / Z$ in Eq. (1), and the Kubo-transformed version ${ }^{112}$ with $\hat{A}_{\text {Kubo }}^{\beta}=\int_{0}^{\beta} \mathrm{d} \lambda e^{-(\beta-\lambda) \hat{H}} \hat{A} e^{-\lambda \hat{H}} / Z \beta$ in Eq. (1). These three versions are related to one another by the following identities between their Fourier transforms

$$
\frac{\beta \hbar \omega}{1-e^{-\beta \hbar \omega}} \underline{C}^{K u b o}(\omega)=\underline{C}(\omega)=e^{\beta \hbar \omega / 2} \underline{C}^{\text {sym }}(\omega)
$$

where the spectrum $\underline{C}(\omega)$ is defined in Eq. (3), etc. Substituting Eq. (A.8) into Eq. (6) leads to the analytic continuation equations for different versions of the autocorrelation functions. For instance, for Kubo-transformed version, one has

$$
C(i \hbar \lambda)=\int_{0}^{\infty} d \omega K^{k u b o}(\lambda, \omega) \underline{C}^{k u b o}(\omega)
$$

where

$$
K^{k u b o}(\lambda, \omega)=\frac{\beta \hbar \omega\left(e^{-\lambda \hbar \omega}+e^{-(\beta-\lambda) \hbar \omega}\right)}{2 \pi\left(1-e^{-\beta \hbar \omega}\right)}
$$

for which similar studies can be found in the literature ${ }^{77,84}$. 


\section{References}

$1 \quad$ W. H. Miller, J. Chem. Phys. 53, 3578 (1970).

W. H. Miller, J. Phys. Chem. A 105, 2942 (2001).

M. Thoss and H. B. Wang, Ann. Rev. Phys. Chem. 55, 299 (2004).

W. H. Miller, Proc. Nat. Acad. Sci. USA. 102, 6660 (2005).

W. H. Miller, J. Chem. Phys. 125, 132305 (2006).

6

J. Liu and W. H. Miller, J. Chem. Phys. 126, 234110 (2007).

M. F. Herman and E. Kluk, Chem. Phys. 91, 27 (1984).

8

E. J. Heller, J. Chem. Phys. 95, 9431 (1991).

E. J. Heller, J. Chem. Phys. 94, 2723 (1991).

G. Campolieti and P. Brumer, J. Chem. Phys. 96, 5969 (1992).

G. Campolieti and P. Brumer, Phys. Rev. A 50, 997 (1994).

K. G. Kay, J. Chem. Phys. 100, 4377 (1994).

K. G. Kay, J. Chem. Phys. 100, 4432 (1994).

K. G. Kay, Ann. Rev. Phys. Chem. 56, 255 (2005).

A. R. Walton and D. E. Manolopoulos, Mol. Phys. 87, 961 (1996).

S. Zhang and E. Pollak, J. Chem. Phys. 119, 11058 (2003).

E. Martin-Fierro and E. Pollak, J. Chem. Phys. 125, 164104 (2006).

W. H. Miller, Adv. Chem. Phys. 25, 69 (1974). 
W. H. Miller, Adv. Chem. Phys. 30, 77 (1975).

R. A. Marcus, J. Chem. Phys. 56, 3548 (1972).

W. H. Miller, Faraday Discuss. 110, 1 (1998).

D. J. Tannor and S. Garashchuk, Annu. Rev. Phys. Chem. 51, 553 (2000).

J. Liu and W. H. Miller, J. Chem. Phys. 125, 224104 (2006).

J. Liu and W. H. Miller, J. Chem. Phys. 127, 114506 (2007).

J. Liu and W. H. Miller, J. Chem. Phys. 128, 144511 (2008).

N. Ananth, C. Venkataraman, and W. H. Miller, J. Chem. Phys. 127, 084114 (2007).

A. Nakayama and N. Makri, J. Chem. Phys. 119, 8592 (2003).

N. Makri, A. Nakayama, and N. Wright, J. Theor. Comp. Chem. 3, 391 (2004).

A. Nakayama and N. Makri, Proc. Nat. Acad Sci. USA 102, 4230 (2005).

B. B. Issack and P. N. Roy, J. Chem. Phys. 126, 024111 (2007).

B. B. Issack and P. N. Roy, J. Chem. Phys. 127, 144306 (2007).

B. B. Issack and P. N. Roy, J. Chem. Phys. 127, 054105 (2007).

Q. Shi and E. Geva, J. Phys. Chem. A 107, 9059 (2003).

Q. Shi and E. Geva, J. Phys. Chem. A 107 (2003).

B. J. Ka, Q. Shi, and E. Geva, J. Phys. Chem. A 109, 5527 (2005).

B. J. Ka and E. Geva, J. Phys. Chem. A 110, 9555 (2006). 
I. Navrotskaya and E. Geva, J. Phys. Chem. A 111, 460 (2007).

X. Sun and W. H. Miller, J. Chem. Phys. 106, 916 (1997).

H. Wang, X. Sun, and W. H. Miller, J. Chem. Phys. 108, 9726 (1998).

X. Sun, H. Wang, and W. H. Miller, J. Chem. Phys. 109, 7064 (1998).

T. Yamamoto, H. B. Wang, and W. H. Miller, J. Chem. Phys. 116, 7335 (2002).

R. Hernandez and G. A. Voth, Chem. Phys. 233, 243 (1998).

J. A. Poulsen, G. Nyman, and P. J. Rossky, J. Chem. Phys. 119, 12179 (2003).

E. P. Wigner, Phys. Rev. 40, 749 (1932).

E. J. Wigner, Trans. Faraday Soc. 34, 29 (1938).

E. J. Heller, J. Chem. Phys. 65, 1289 (1976).

H. W. Lee and M. O. Scully, J. Chem. Phys. 73, 2238 (1980).

E. Pollak and J. L. Liao, J. Chem. Phys. 108, 2733 (1998).

Q. Shi and E. Geva, J. Chem. Phys. 118, 8173 (2003).

J. Shao and N. Makri, J. Phys. Chem. A 103, 7753 (1999).

J. Shao and N. Makri, J. Phys. Chem. A 103, 9479 (1999).

N. Makri, J. Phys. Chem. B 106, 8390 (2002).

N. J. Wright and N. Makri, J. Chem. Phys. 119, 1634 (2003).

N. J. Wright and N. Makri, J. Phys. Chem. B 108, 6816 (2004).

A. Nakayama and N. Makri, Chem. Phys. 304, 147 (2004). 
J. Liu and N. Makri, Chem. Phys. 322, 23 (2006).

J. Liu, A. Nakayama, and N. Makri, Mol. Phys. 104, 1267 (2006).

J. Kegerreis and N. Makri, J. Comput. Chem. 28, 818 (2007).

E. Bukhman and N. Makri, J. Phys. Chem. A 111, 11320 (2007).

J. Chen and N. Makri, Mol. Phys. 106, 443 (2008).

J. Kegerreis, A. Nakayama, and N. Makri, J. Chem. Phys. 128, 184509 (2008).

X. Sun and W. H. Miller, J. Chem. Phys. 110, 6635 (1999).

M. Thoss, H. Wang, and W. H. Miller, J. Chem. Phys. 114, 9220 (2001).

J. A. Poulsen, G. Nyman, and P. J. Rossky, J. Phys. Chem. B 108, 19799 (2004).

J. A. Poulsen, G. Nyman, and P. J. Rossky, J. Phys. Chem. A 108, 8743 (2004).

T. D. Hone, J. A. Poulsen, P. J. Rossky, and D. E. Manolopoulos, J. Phys. Chem. B 112, 294 (2008).

R. N. Silver, D. S. Sivia, and J. E. Gubernatis, Phys. Rev. B 41, 2380 (1990).

J. E. Gubernatis, M. Jarrell, R. N. Silver, and D. S. Sivia, Phys. Rev. B 44, 6011 (1991).

M. Jarrell and J. E. Gubernatis, Phys. Rep. 269, 133 (1996).

M. Boninsegni and D. M. Ceperley, J. Low Temp. Phys. 104, 339 (1996).

D. Kim, J. D. Doll, and J. E. Gubernatis, J. Chem. Phys. 106, 1641 (1997).

E. Galliccio and B. J. Berne, J. Chem. Phys. 101, 9909 (1994).

E. Galliccio and B. J. Berne, J. Chem. Phys. 105, 7064 (1996). 
S. A. Egorov, E. Gallicchio, and B. J. Berne, J. Chem. Phys. 107, 9312 (1997).

E. Gallicchio, S. A. Egorov, and B. J. Berne, J. Chem. Phys. 109, 7745 (1998).

G. Krilov and B. J. Berne, J. Chem. Phys. 111, 9147 (1999).

E. Rabani, G. Krilov, and B. J. Berne, J. Chem. Phys. 112, 2605 (2000).

G. Krilov, E. Sim, and B. J. Berne, Chem. Phys. 268, 21 (2001).

E. Sim, G. Krilov, and B. J. Berne, J. Phys. Chem. A 105, 2824 (2001).

G. Krilov, E. Sim, and B. J. Berne, J. Chem. Phys. 114, 1075 (2001).

E. Rabani, D. R. Reichman, G. Krilov, and B. J. Berne, Proc. Nat. Acad. Sci. USA 99, 1129 (2002).

R. E. Zillich, F. Paesani, Y. Kwon, and K. B. Whaley, J. Chem. Phys. 123 (2005).

S. Habershon, B. J. Braams, and D. E. Manolopoulos, J. Chem. Phys. 127, 174108 (2007).

I. R. Craig and D. E. Manolopoulos, J. Chem. Phys. 121, 3368 (2004).

I. R. Craig and D. E. Manolopoulos, J. Chem. Phys. 122, 084106 (2005).

I. R. Craig and D. E. Manolopoulos, Chem. Phys. 322, 236 (2006).

T. F. Miller and D. E. Manolopoulos, J. Chem. Phys. 123, 154504 (2005).

T. F. Miller and D. E. Manolopoulos, J. Chem. Phys. 122, 184503 (2005).

B. J. Braams and D. E. Manolopoulos, J. Chem. Phys. 125 (2006).

R. Collepardo-Guevara, I. R. Craig, and D. E. Manolopoulos, J. Chem. Phys. 128 (2008).

T. E. Markland, S. Habershon, and D. E. Manolopoulos, J. Chem. Phys. 128 (2008). 
J. Cao and G. A. Voth, J. Chem. Phys. 99, 10070 (1993).

J. Cao and G. A. Voth, J. Chem. Phys. 101, 6157 (1994).

J. Cao and G. A. Voth, J. Chem. Phys. 101, 6168 (1994).

J. Cao and G. A. Voth, J. Chem. Phys. 100, 5106 (1994).

J. Cao and G. A. Voth, J. Chem. Phys. 101, 6168 (1994).

J. Cao and G. A. Voth, J. Chem. Phys. 101, 6184 (1994).

S. Jang and G. A. Voth, J. Chem. Phys. 111, 2371 (1999).

S. Jang and G. A. Voth, J. Chem. Phys. 111, 2357 (1999).

D. R. Reichman, P. N. Roy, S. Jang, and G. A. Voth, J. Chem. Phys. 113, 919 (2000).

T. D. Hone and G. A. Voth, J. Chem. Phys. 121, 6412 (2004).

F. Paesani, S. Luchi, and G. A. Voth, J. Chem. Phys. 127, 074506 (2007).

K. Kinugawa, Chem. Phys. Lett. 292, 454 (1998).

K. Kinugawa, H. Nagao, and K. Ohta, J. Chem. Phys. 114, 1454 (2001).

Q. Shi and E. Geva, J. Chem. Phys. 119, 9030 (2003).

E. Rabani and D. R. Reichman, Annu. Rev. Phys. Chem. 56, 157 (2005).

T. F. Miller and D. Chandler, (submitted).

G. Baym and N. D. Mermin, J. Math. Phys. 2, 232 (1961).

B. J. Berne and G. D. Harp, Adv. Chem. Phys. 17, 63 (1970).

W. H. Miller, S. D. Schwartz, and J. W. Tromp, J. Chem. Phys. 79, 4889 (1983). 

Mechanics, 2nd ed. (Springer-Verlag, Heidelberg, 1991).

K. Yamashita and W. H. Miller, J. Chem. Phys. 82, 5475 (1985).

W. H. Press, S. A. Teukolsky, W. T. Vetterling, and B. P. Flannery, Numerical Recipes in Fortran 77, Second ed. (Cambridge University Press, Cambridge, 1992). Academic, Dortrecht, 1989). Academic, Dortrecht, 1989). NJ, 1974). 
P. A. Frantsuzov and V. A. Mandelshtam, Phys. Rev. E 72, 37102 (2005).

N. Makri and K. Thompson, Chem. Phys. Lett. 291, 101 (1998).

K. Thompson and N. Makri, Phys. Rev. E 59, R4729 (1999).

However, one should not read too much into the comparison with experiments for this system, for reasons discussed in Refs. 25 and 84. . function at zero time $(\mathrm{t}=0)$ since the classical dynamics fails to do so for general correlation functions. B. N. Esel'son, Y. P. Blagoi, V. V. Grigor'ev, V. G. Manzhelii, S. A. Mikhailenko, and N. P. Neklyudov, Properties of Liquid and Solid Hydrogen. (Israel Program for Scientific Translations, Jerusalem, 1971). 
Table. 1 Diffusion contants for liquid para-hydrogen at $T=25 \mathrm{~K}, v=31.7 \mathrm{~cm}^{3} \mathrm{~mol}^{-1}$ under nearly zero extent pressure

\begin{tabular}{|c|c|c|c|}
\hline & \multirow[b]{2}{*}{$N_{p}$} & \multicolumn{2}{|c|}{ Diffusion constant $\left(\AA^{2} / \mathrm{ps}\right)$ at $25 \mathrm{~K}$} \\
\hline & & prior & MEAC correction \\
\hline Experiment $^{138}$ & & 1.6 & \\
\hline TGA/LSC-IVR & $108^{*}$ & $1.81^{25}$ & 1.80 \\
\hline RPMD & 256 & $1.44^{84}$ & $1.78^{84}$ \\
\hline CMD & 216 & $1.39^{102}$ & 1.70 \\
\hline \multirow[t]{2}{*}{ Classical } & 108 & 1.52 & 1.87 \\
\hline & $\rightarrow \infty$ & $0.56^{89}$ & -- \\
\hline Flat prior & 108 & -- & 1.46 \\
\hline
\end{tabular}

* We also extend the system size to 216 molecules per box and the results are nearly the same. But how the diffusion constant goes in the thermal dynamic limit $\left(N_{p} \rightarrow \infty\right.$ ) for all MEAC corrections still needs further investigation. 
Table. 2 Diffusion contants for liquid para-hydrogen at $T=14 \mathrm{~K} ; v=25.6 \mathrm{~cm}^{3} \mathrm{~mol}^{-1}$ under nearly zero extent pressure

\begin{tabular}{llll}
\hline \hline & & \multicolumn{2}{c}{ Diffusion constant $\left(\AA^{2} / \mathrm{ps}\right)$ at $14 \mathrm{~K}$} \\
\cline { 3 - 4 } & $N_{p}$ & prior & MEAC correction \\
\hline Experiment $^{138}$ & $108^{*}$ & 0.4 & 0.47 \\
TGA/LSC-IVR & 256 & $0.63^{25}$ & $0.41^{84}$ \\
RPMD & 216 & $0.27^{84}$ & 0.37 \\
CMD & 108 & $0.34^{102}$ & 0.34 \\
Classical & $\rightarrow \infty$ & 0.26 & - \\
Flat prior & 108 & $0.02^{89}$ & 0.43 \\
\hline \hline
\end{tabular}




\section{Figure Captions}

Fig. 1 (Color online). Imaginary time autocorrelation function $\left\langle\exp \left[-5 x(0)^{2}\right] \exp \left[-5 x(i \hbar \lambda)^{2}\right]\right\rangle$ for the harmonic oscillator $V(x)=0.5 x^{2}$ with the mass $m=1$ and $\hbar=1$ at the inverse temperature $\beta=5$. Solid Line: Exact results. Hollow Cycles: the MEAC using the flat prior with the "L curve" technique (MEAC-flat-L). Solid Triangles: the MEAC using the flat prior with the classical maximum entropy (CME) technique (MEAC-flat-CME).

Fig. 2 (Color online). Comparison of the LSC-IVR to the MEAC-flat for the standard real time autocorrelation function $\left\langle\exp \left[-5 x(0)^{2}\right] \exp \left[-5 x(t)^{2}\right]\right\rangle$ for the harmonic oscillator $V(x)=0.5 x^{2}$ with the mass $m=1$ and $\hbar=1$ at the inverse temperature $\beta=5$. Panel (b) shows a blowup of the curves shown in (a).

Fig. 3 (Color online). Comparison of the LSC-IVR to the MEAC-flat at a relatively high temperature $\beta=1$ for the quartic potential $V(x)=0.25 x^{4}$ with the mass $m=1$ and $\hbar=1$ : (a) Standard momentum autocorrelation function (divided by $2 \mathrm{~m}$ ) and (b) Kubo-transformed force autocorrelation function, the force $f(x)=-V^{\prime}(x)=-x^{3}$

Fig. 4 (Color online). Comparison of imaginary time data transformed from corresponding real time autocorrelation functions based on the LSC-IVR to those based on the MEAC+LSC/IVR, for the quartic potential at the inverse temperatures $\beta=1$ : (a) the imaginary momentum autocorrelation function (divided by $2 m$ ) $\langle p(0) p(i \hbar \lambda)\rangle / 2 m$ and (b) the imaginary force autocorrelation function $\langle f(0) f(i \hbar \lambda)\rangle$. Solid Line: Exact results. Hollow Cycles: the MEAC using the LSC-IVR prior with the "L curve" technique (MEAC-LSC/IVR-L). Solid Triangles: the MEAC using the LSC-IVR prior with the CME technique 
(MEAC-LSC/IVR-CME). Long-dashed line: the LSC-IVR.

Fig. 5 (Color online). Comparison of the standard momentum autocorrelation function (divided by $2 \mathrm{~m}$ ) $\langle p(0) p(t)\rangle / 2 m$ [Panel (a)] and the Kubo-transformed force autocorrelation function $\langle f(0) f(t)\rangle_{k u b o}$ [Panel (b)] from the LSC-IVR to those from the MEAC+LSC/IVR for the quartic potential at the inverse temperature $\beta=1$.

Fig. 6 (Color online). Same as in Fig. 4, but at the inverse temperature $\beta=10$.

Fig. 7 (Color online). Same as in Fig. 5, but at the inverse temperature $\beta=10$.

Fig. 8 (Color online). The imaginary momentum autocorrelation function per particle (divided by $m^{2}$ ) transformed from the corresponding Kubo-transformed real time correlation function from different trajectory-based approaches (LSC-IVR, RPMD, CMD and classical dynamics) for liquid para- $\mathrm{H}_{2}$ at two state points: (a) $T=25 \mathrm{~K}, v=31.7 \mathrm{~cm}^{3} \mathrm{~mol}^{-1}$ and (b) $T=14 \mathrm{~K} ; v=25.6 \mathrm{~cm}^{3} \mathrm{~mol}^{-1}$.

Fig. 9 (Color online). Spectra of Kubo-transformed momentum autocorrelation functions for the liquid para- $\mathrm{H}_{2}$ at the state point $T=25 \mathrm{~K}, v=31.7 \mathrm{~cm}^{3} \mathrm{~mol}^{-1}$. Comparison among different trajectory-based approaches [Panel (a)], comparison among the MEAC results with different priors [Panel (b)], and comparisons of each prior to its MEAC correction [Panels (c-f)].

Fig. 10 (Color online). Same as in Fig. 9, but at the different state point $T=14 \mathrm{~K} ; v=25.6 \mathrm{~cm}^{3} \mathrm{~mol}^{-1}$.

Fig. 11 (Color online). Kubo-transformed momentum autocorrelation functions (divided by $2 m k_{B}$ ) for the liquid para $-\mathrm{H}_{2}$ at the state point $T=25 \mathrm{~K}, v=31.7 \mathrm{~cm}^{3} \mathrm{~mol}^{-1}$. Comparison among different trajectory-based approaches [Panel (a)], comparison among the MEAC results with different priors [Panel (b)], and comparisons 
between the LSC-IVR (and its MEAC-corrected version) to each of other trajectory-based approaches (and their MEAC corrections) [Panels (c-e)].

Fig. 12 (Color online). Same as in Fig. 11, but at the different state point $T=14 \mathrm{~K} ; v=25.6 \mathrm{~cm}^{3} \mathrm{~mol}^{-1}$.

Fig. 13 (Color online). $g^{R}(\lambda, t)$ in Eq. (A.6) as a function of $t$ for $\lambda / \beta=1 / 2,1 / 4,1 / 8$, and $1 / 16$, which illustrates the degree to which the imaginary time data $C(i \hbar \lambda)$ constrains the real time data and vice versa. Notice that $g^{R}(\lambda, t)$ goes to a delta function $\delta(t)$ as $\lambda \rightarrow 0$ and $g^{R}(\lambda, t)=g^{R}(\beta-\lambda, t)$.

Fig. 14 (Color online). The function $G^{R}\left(\lambda ; t_{1}, t_{2}\right)$ defined in Eq. (A.7) as a function of $\lambda$, which demonstrates how the imaginary time data $C(i \hbar \lambda)$ affect the real time data in the interval $\left[t_{1}, t_{2}\right]$ and vice versa. 


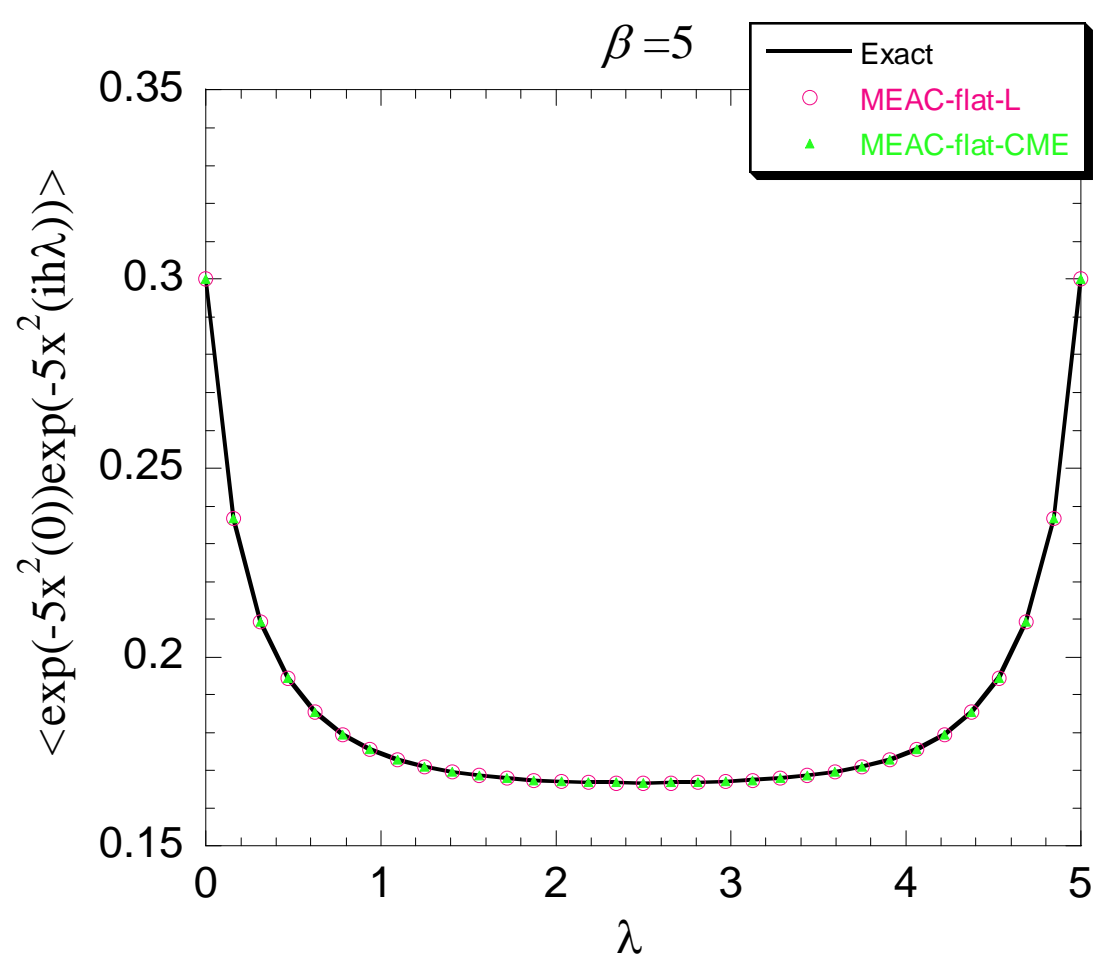

Fig. 1 

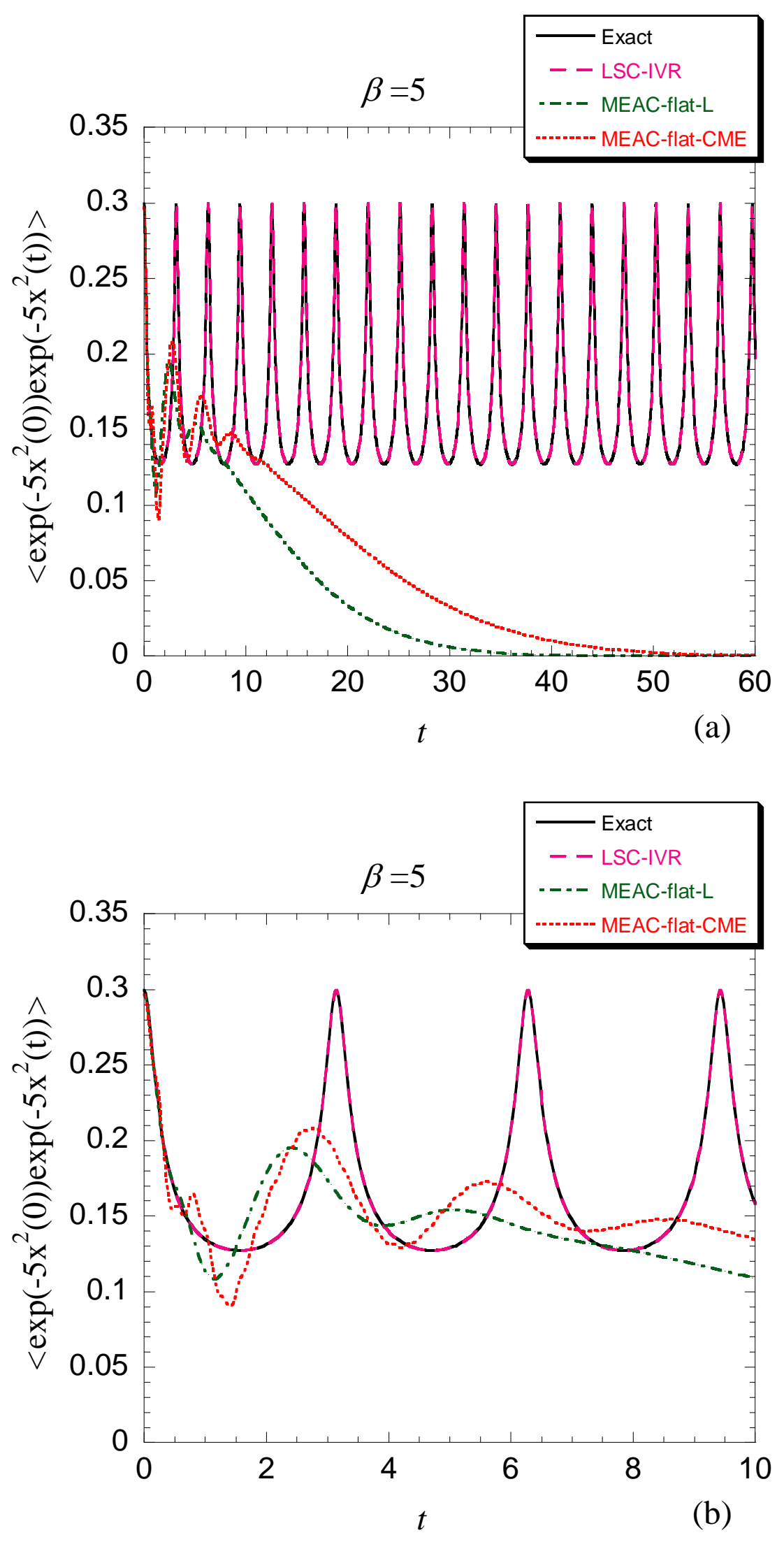

Fig. 2 

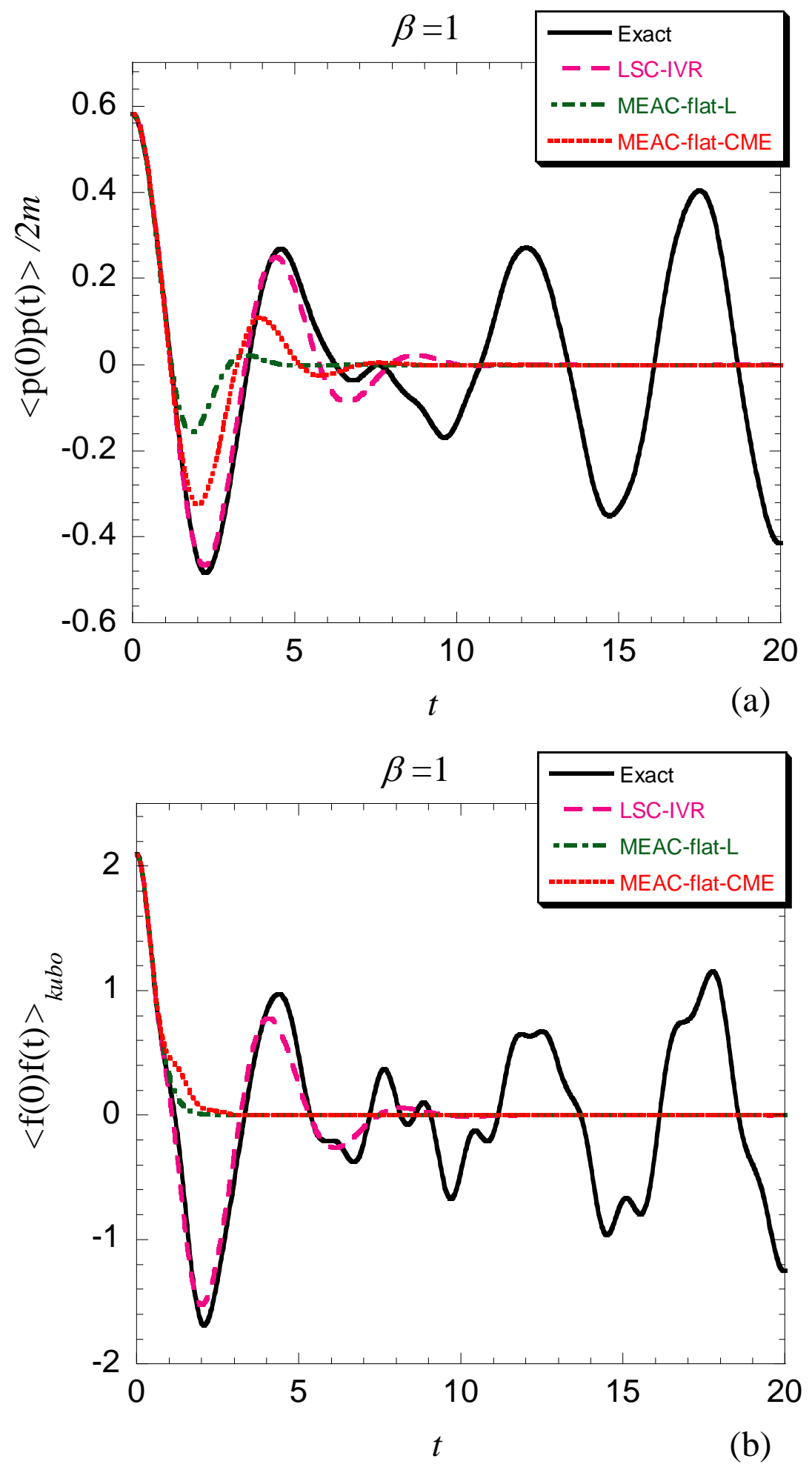

Fig. 3 

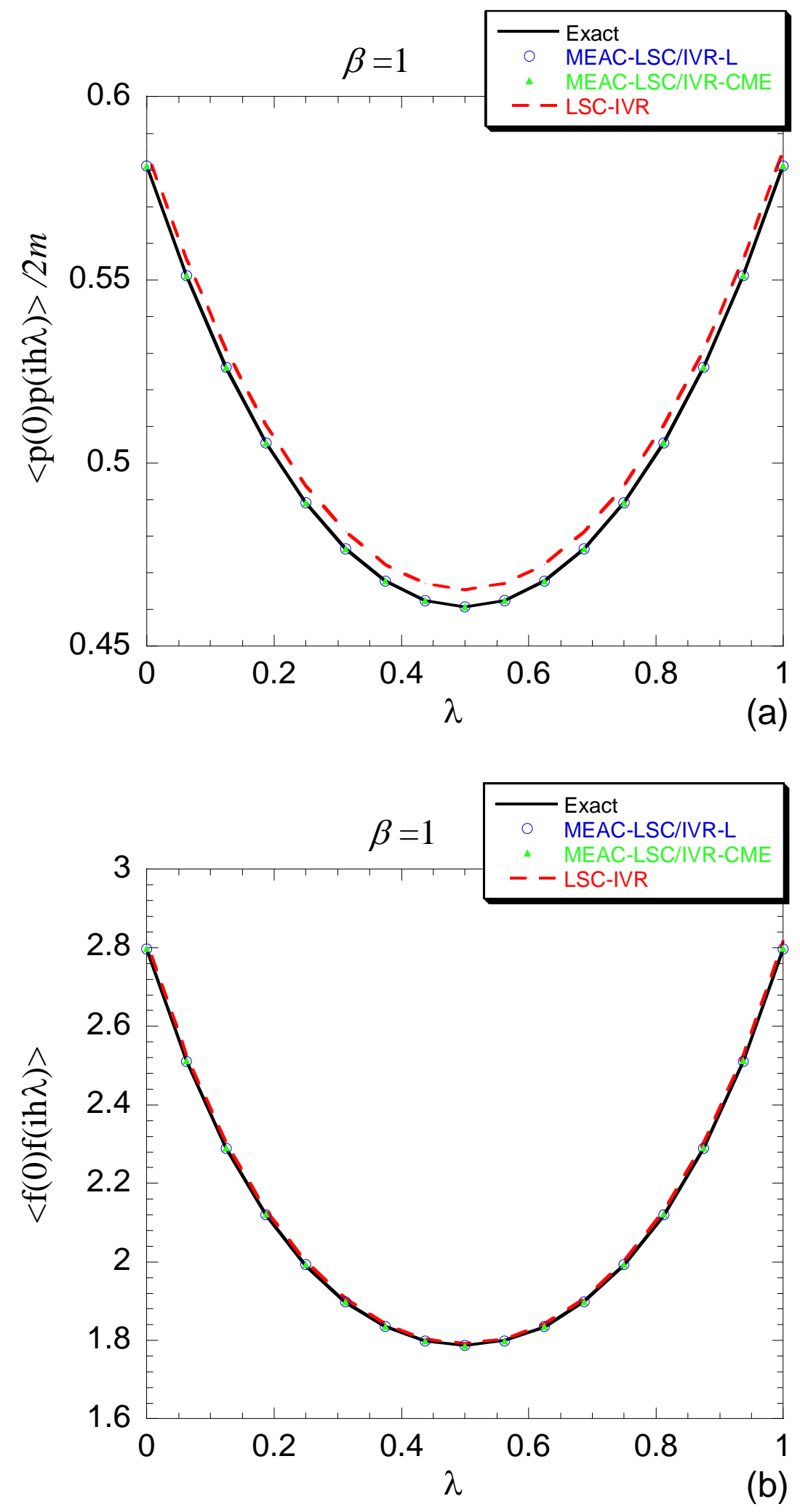

Fig. 4 

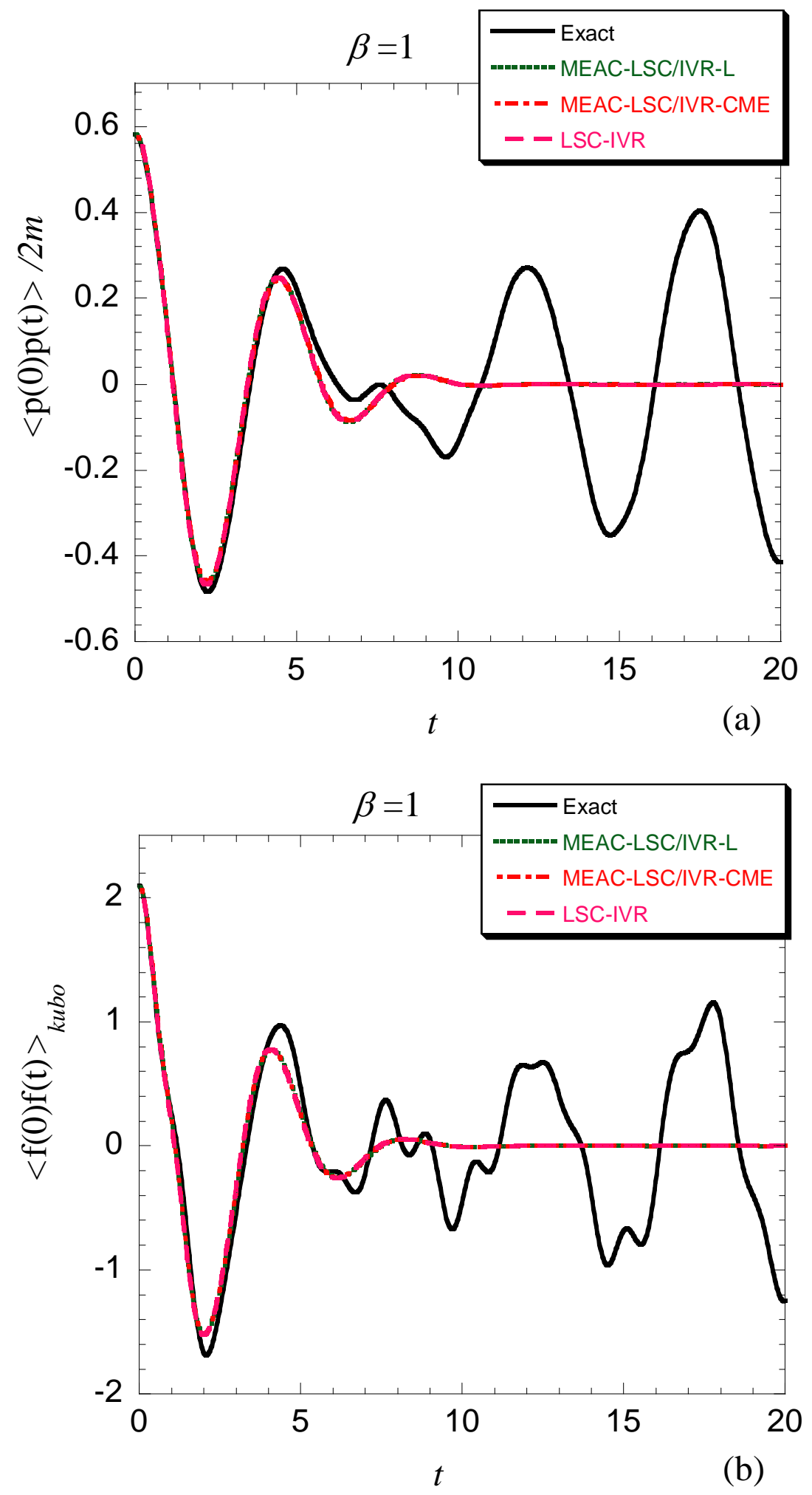

Fig. 5 

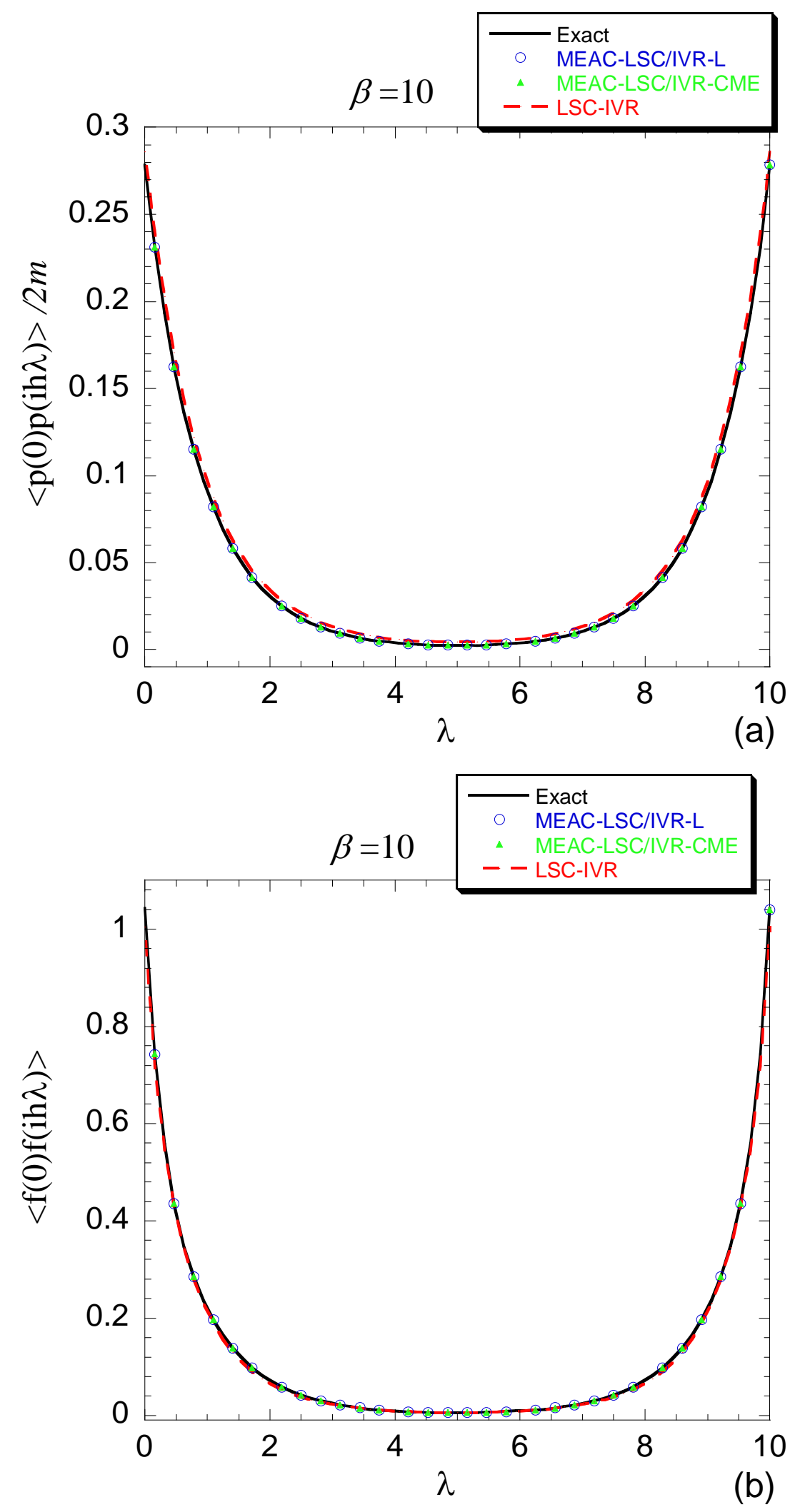

Fig. 6 

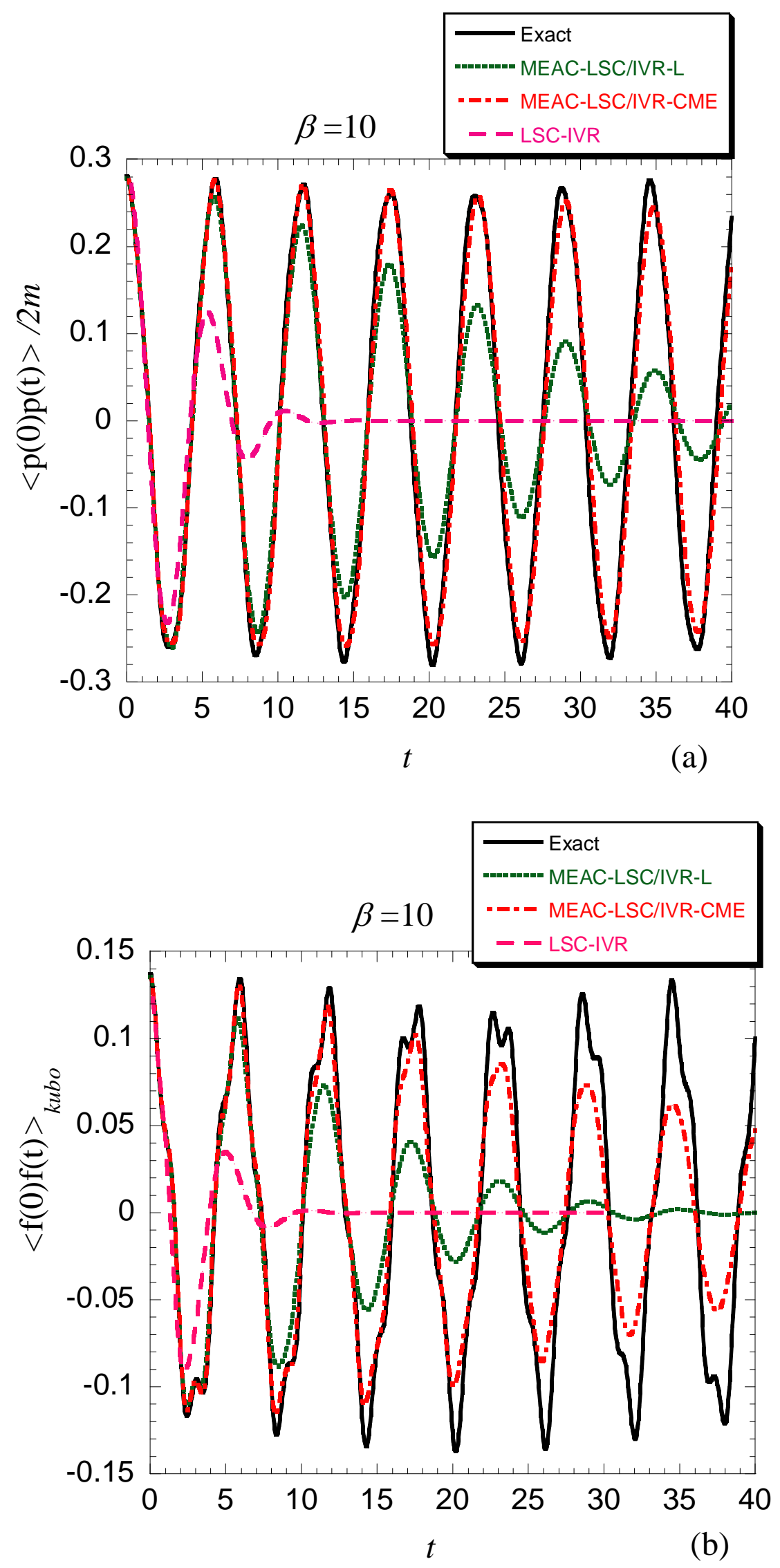

Fig. 7 

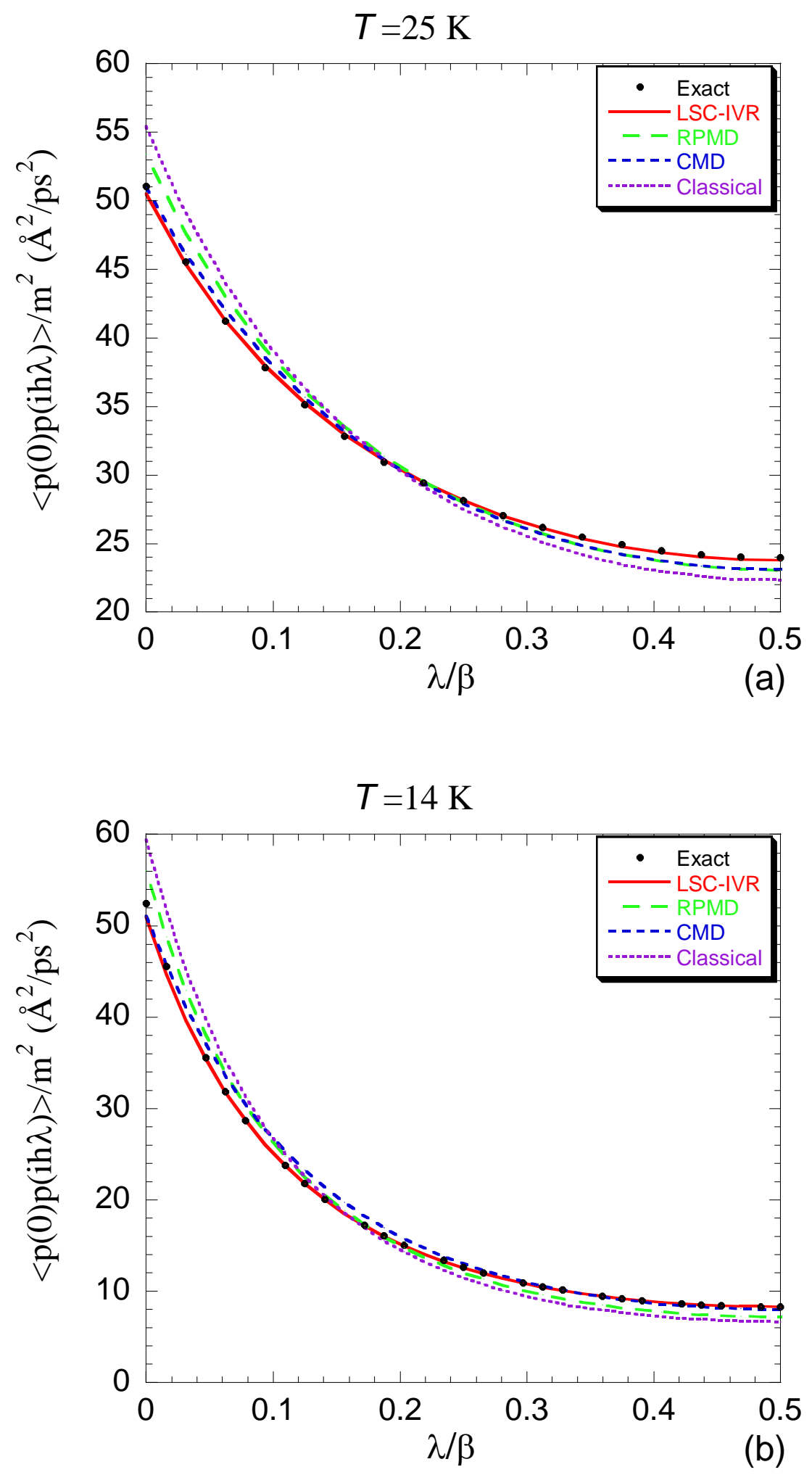

Fig. 8 
$25 \mathrm{~K}$
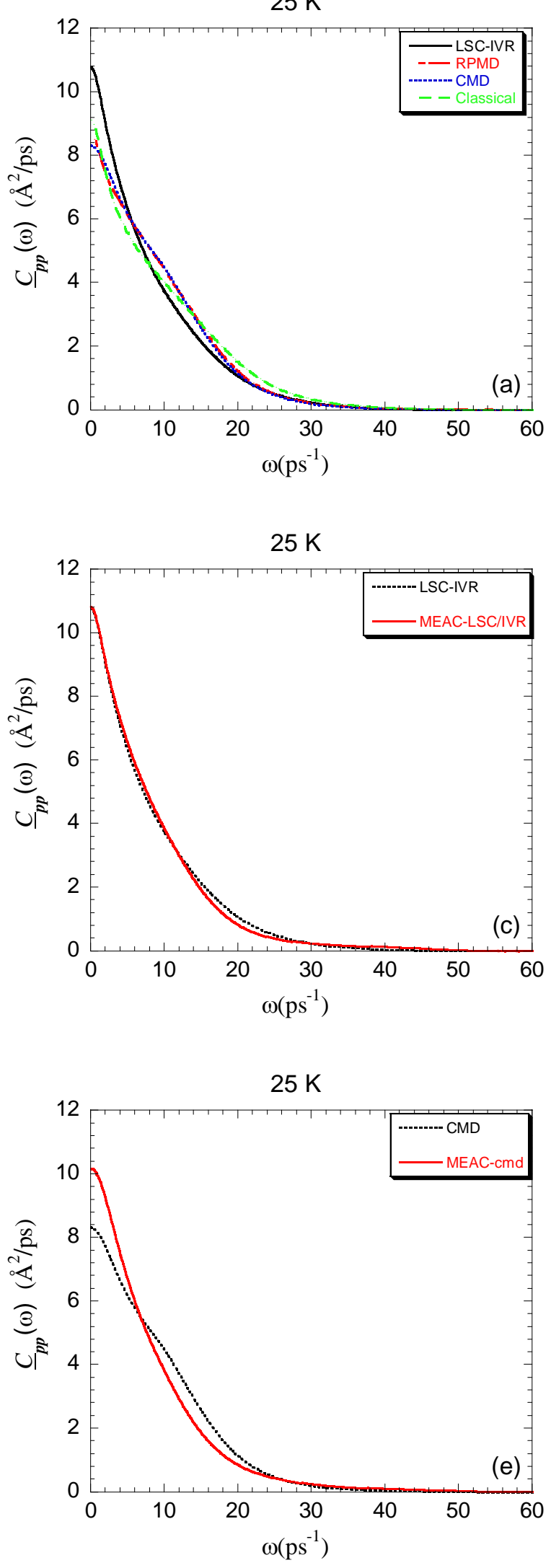
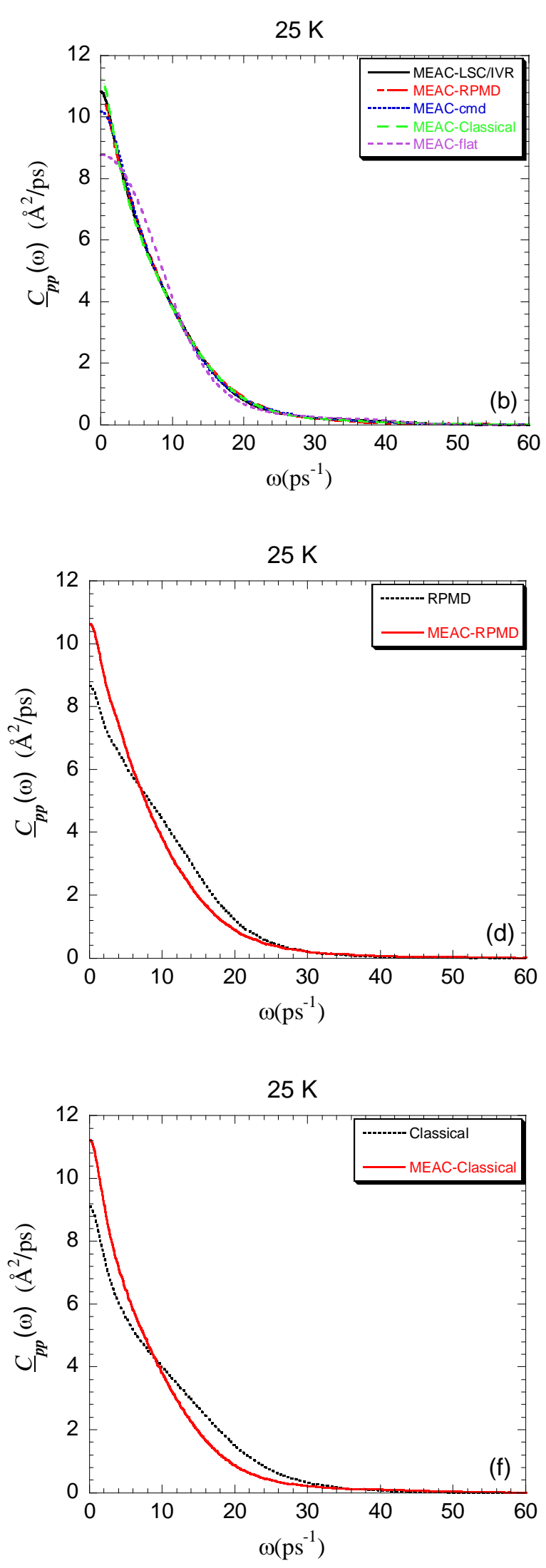

Fig. 9 

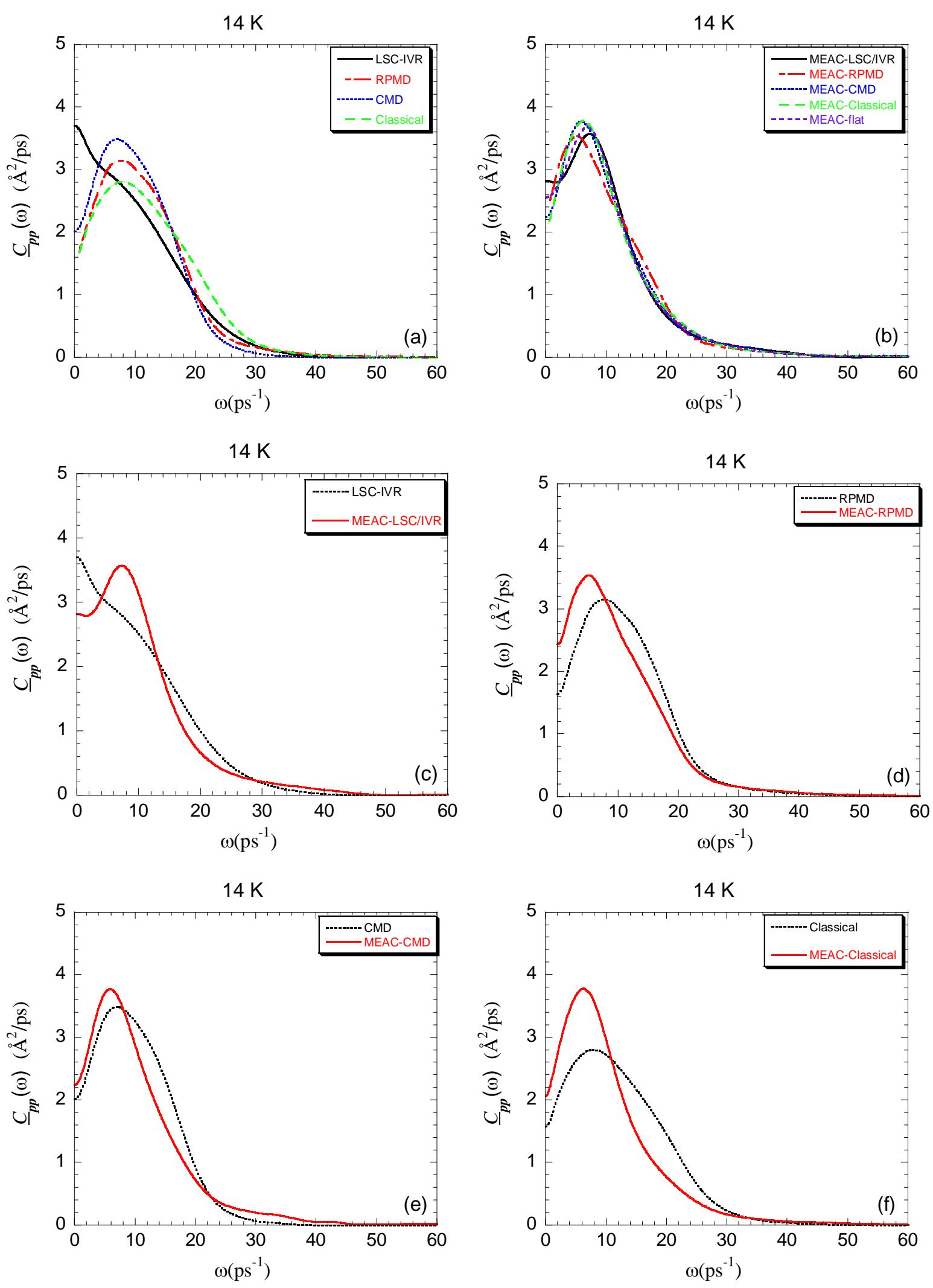

Fig. 10 

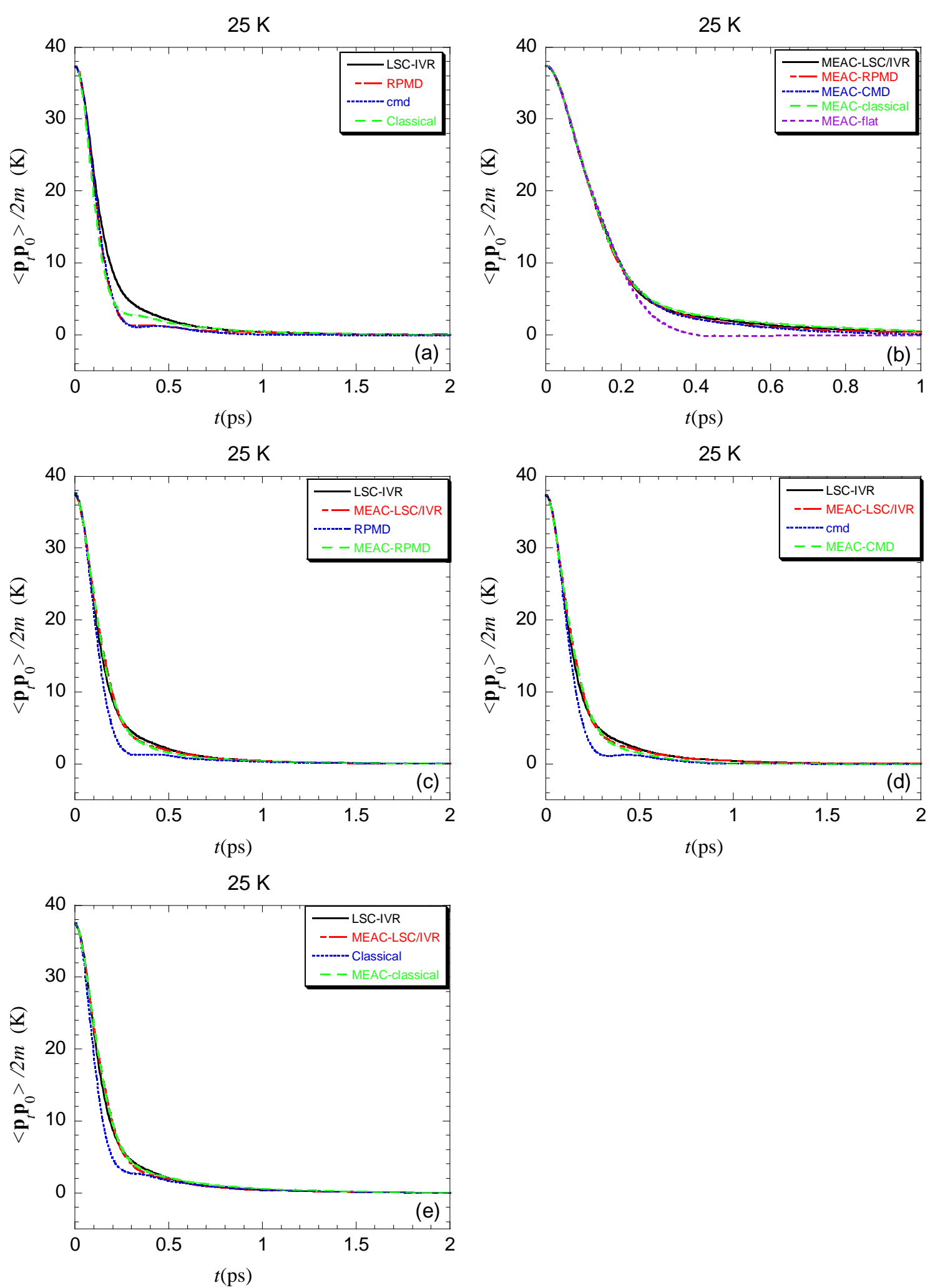

Fig. 11 
$14 \mathrm{~K}$

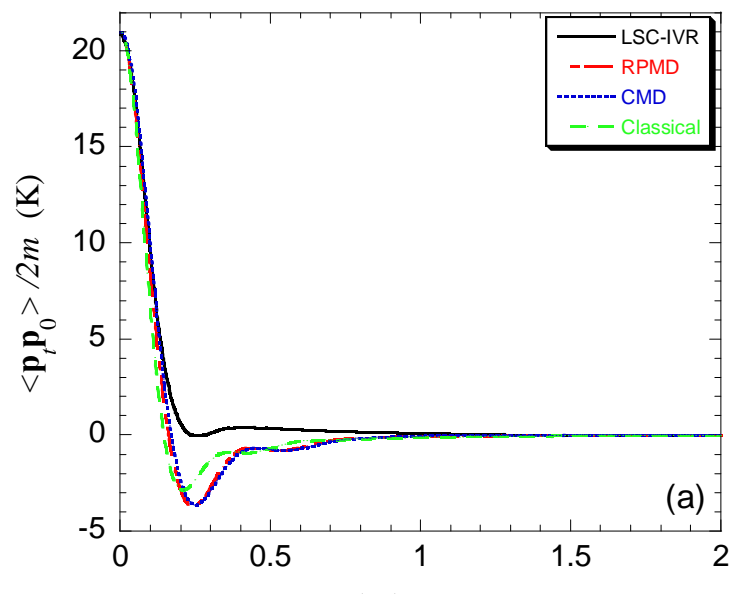

$t$ (ps)

$14 \mathrm{~K}$

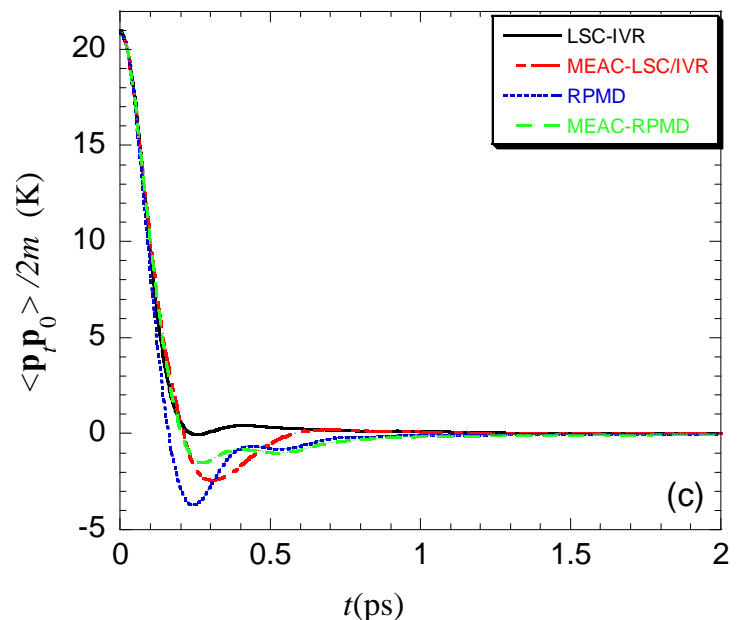

$14 \mathrm{~K}$

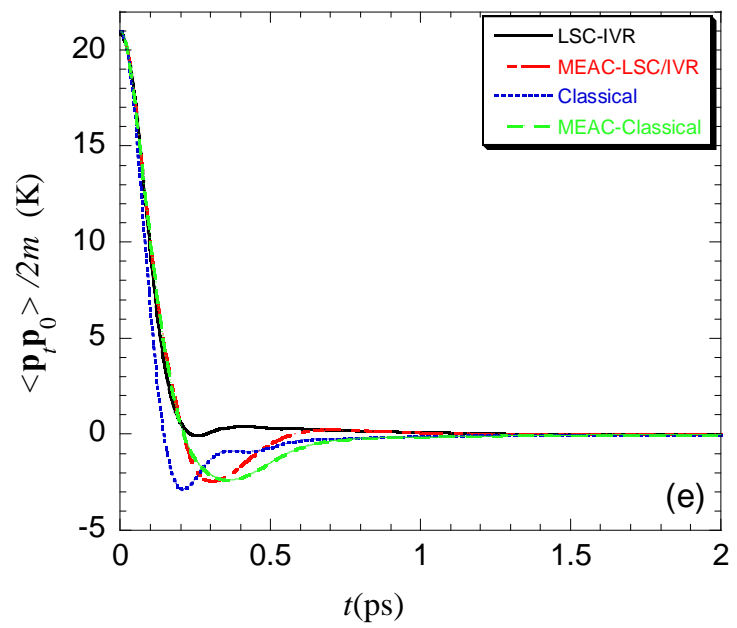

$14 \mathrm{~K}$

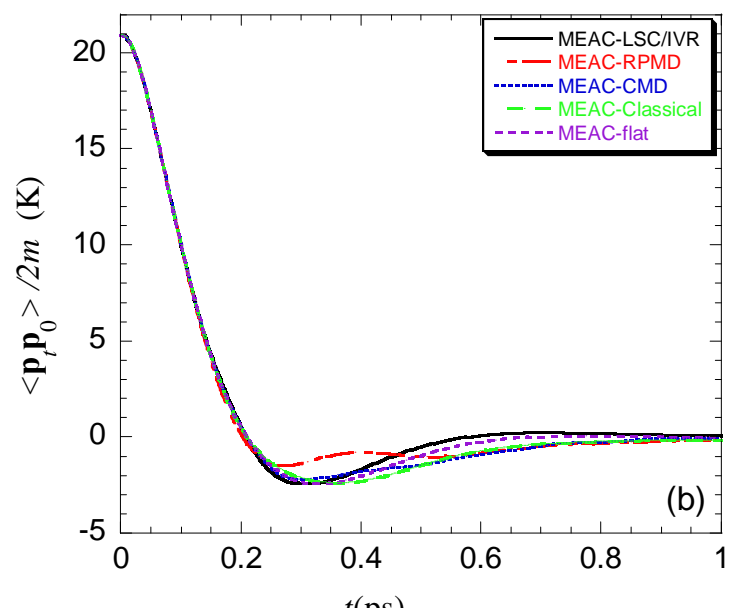

(ps)

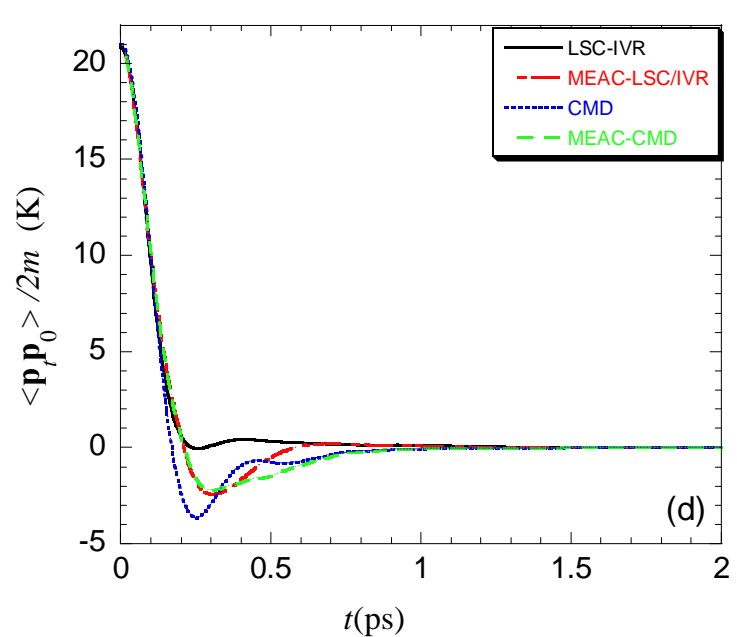

Fig. 12 


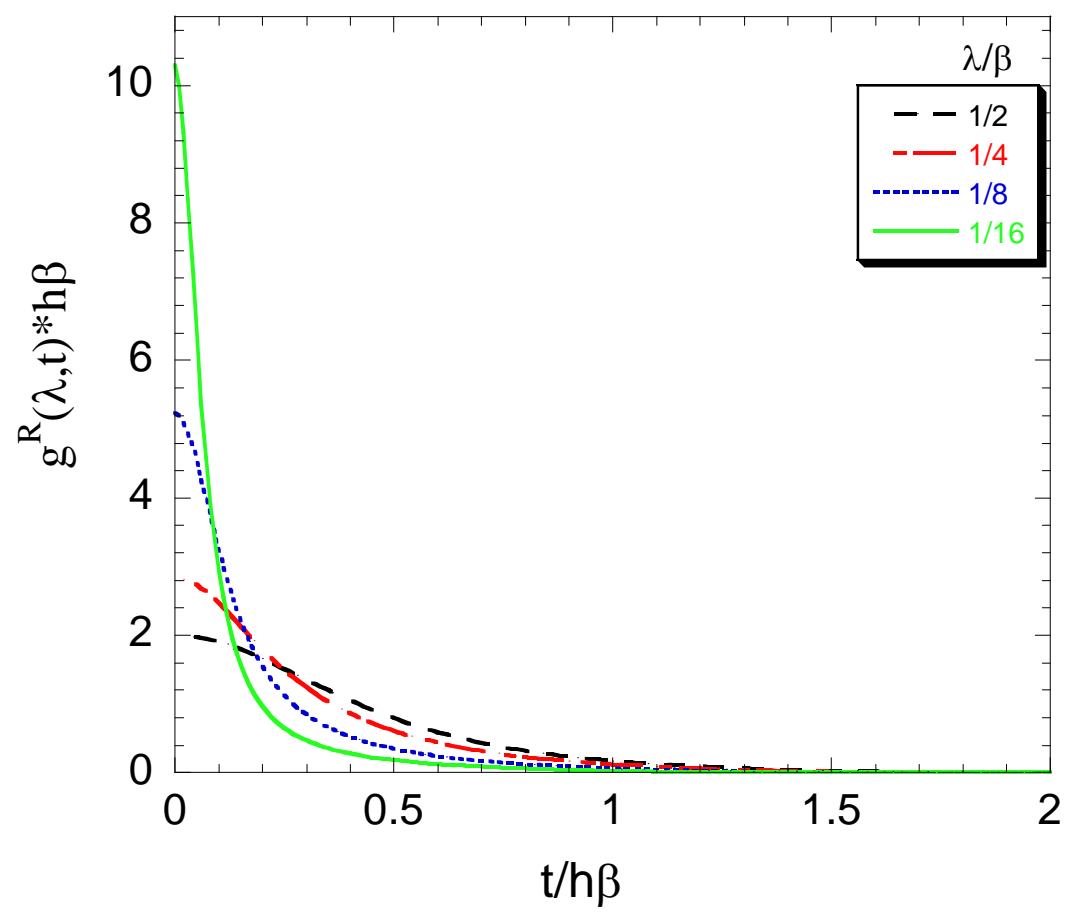

Fig. 13 


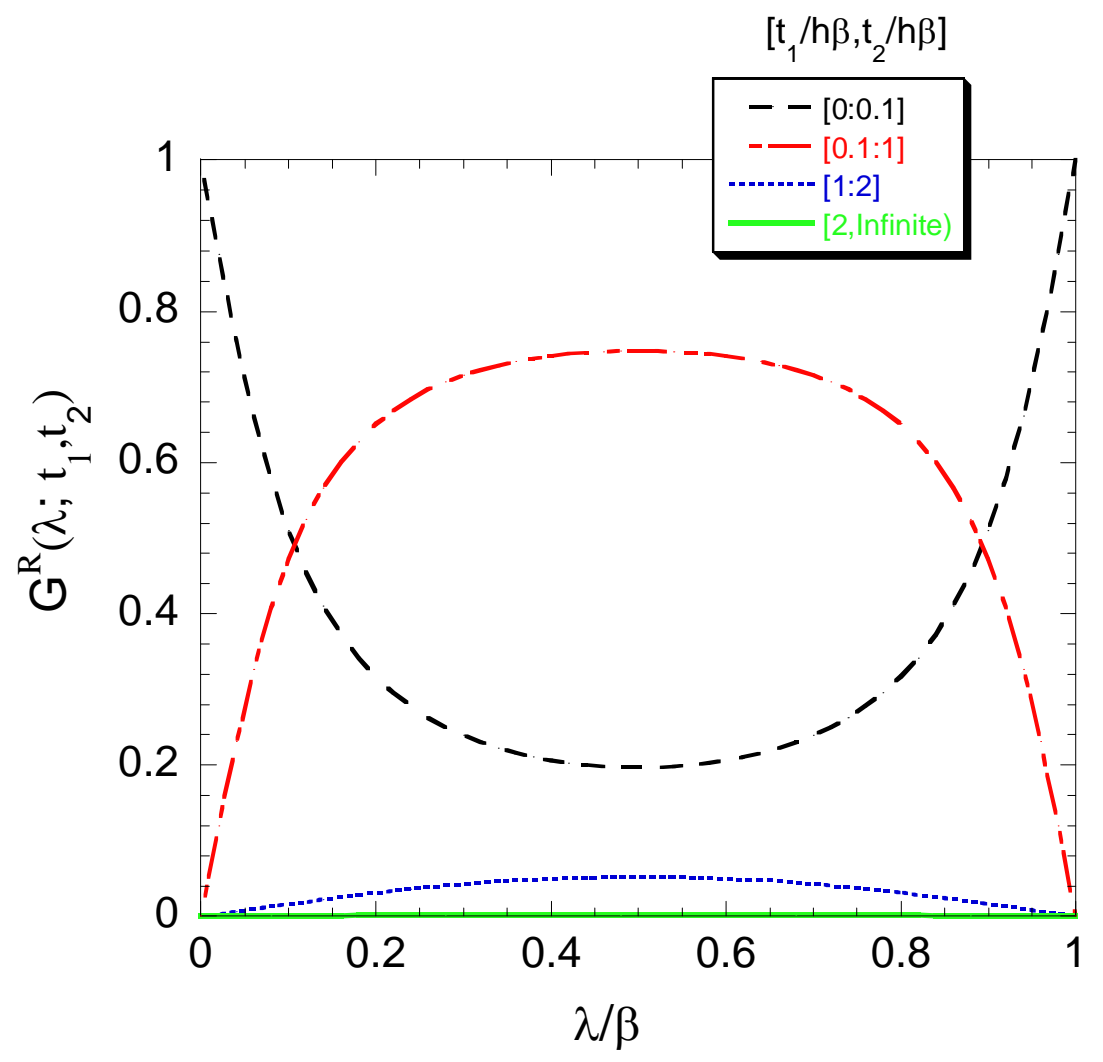

Fig. 14 